\title{
Development and control of a robotic exoskeleton for shoulder, elbow and forearm movement assistance
}

\author{
Mohammad Habibur Rahman a,*, Thierry Kittel-Ouimet ${ }^{\mathrm{a}}$, Maarouf Saad ${ }^{\mathrm{a}}$, Jean-Pierre Kenné \\ and Philippe S. Archambault ${ }^{\mathrm{c}, \mathrm{d}}$ \\ ${ }^{a}$ Department of Electrical Engineering, École de Technologie Supérieure, Montreal, QC, Canada \\ ${ }^{\mathrm{b}}$ Department of Mechanical Engineering, École de Technologie Supérieure, Montreal, QC, Canada \\ ${ }^{\mathrm{c} S}$ Shool of Physical and Occupational Therapy, McGill University, Montreal, QC, Canada \\ ${ }^{\mathrm{d}}$ Centre for Interdisciplinary Research in Rehabilitation, Jewish rehabilitation Hospital, Laval, QC, Canada
}

\begin{abstract}
World health organization reports, annually more than 15 million people worldwide suffer a stroke and cardiovascular disease, among which $85 \%$ of stroke patients incur acute arm impairment, and $40 \%$ of victims are chronically impaired or permanently disabled. This results a burden on the families, communities and to the country as well. Rehabilitation programs are the main way to promote functional recovery in these individuals. Since the number of such cases is constantly growing and that the duration of treatment is long, an intelligent robot could significantly contribute to the success of these programs. We therefore developed a new 5DoFs robotic exoskeleton named MARSE-5 (motion assistive robotic-exoskeleton for superior extremity) that supposed to be worn on the lateral side of upper arm to rehabilitate and ease the shoulder, elbow and forearm movements. This paper focused on the design, modeling, development and control of the proposed MARSE-5. To control the exoskeleton, a nonlinear sliding mode control (SMC) technique was employed. In experiments, trajectory tracking that corresponds to typical passive rehabilitation exercises was carried out. Experimental results reveal that the controller is able to maneuver the MARSE-5 efficiently to track the desired trajectories.
\end{abstract}

Keywords: Arm impairment, passive rehabilitation therapy, robotic exoskeleton, rehabilitation, sliding mode control

\section{Introduction}

Superior extremity impairment such as full or partial loss of upper limb function is a common in the adults, and elderly due to strokes, trauma, sports injuries, occupational injuries, and spinal cord injuries. In addition to these, arm impairment e.g., dislocation of shoulder and/or elbow and/or wrist joint is very

\footnotetext{
${ }^{*}$ Corresponding author: M.H. Rahman, Electrical Engineering Department, GREPCI, (Room \# A-2612), École de Technologie Supérieure, 1100 rue Notre-dame Ouest, Montreal, H3C-1K3, Canada. Tel.: +1 5143968800 Ext. 7982; Fax: +1 514396 8684; E-mail: mhrahman@ieee.org.
}

common in children and also in adults due to sports, falls, and traumatic injuries such as car crash $[9,23$, 33]. To perform essential daily activities, the movements of the shoulder, elbow, and wrist play a vital role and necessitate proper functioning of the superior extremity. Recent studies revealed that virtual reality based rehabilitation and robot-assisted therapy were able to reduce arm impairment $[3,28]$ and significantly improve motor function to regain upper-limb mobility [6]. As first step, therefore, we developed a 2 DoFs robotic-exoskeleton for assisting wrist joint movements [32]. Later on, we have developed another exoskeleton module to rehabilitate and ease elbow 
Table 1

A brief comparison of existing rehabilitative devices/robots

\begin{tabular}{|c|c|c|c|c|c|c|c|}
\hline \multicolumn{8}{|c|}{$\begin{array}{l}\text { Type-1 Exoskeleton type: These are kind of wearable robots or powered orthosis which are designed to be worn on the lateral side of the } \\
\text { upper arm }\end{array}$} \\
\hline Name/year & DoFs & Placement & Purpose & $\begin{array}{c}\text { Clinical } \\
\text { trial }\end{array}$ & Actuator & $\begin{array}{l}\text { Motion } \\
\text { support }\end{array}$ & $\begin{array}{l}\text { Control } \\
\text { method }\end{array}$ \\
\hline MARSE-5, 2012 & 5 & FM & $\mathrm{R}, \mathrm{MA}$ & No & BLDCM & $\mathrm{S}, \mathrm{E}, \mathrm{F}$ & $\mathrm{SMC}$ \\
\hline ExoRob, $2011[29,32]$ & 4 & FM & $\mathrm{R}, \mathrm{MA}$ & No & BLDCM & $\mathrm{E}, \mathrm{W}$ & PID, CTC, SMC \\
\hline Hand motion assist robot [18] & 18 & DM & $\mathrm{R}$ & Yes & EM & $\mathrm{W}, \mathrm{H}$ & $\mathrm{PD}$ \\
\hline ABLE, $2008[11]$ & 4 & FM & $\mathrm{PA}, \mathrm{R}, \mathrm{T}, \mathrm{H}$ & No & DCM & $\mathrm{S}, \mathrm{E}$ & FF \\
\hline MEDARM, 2007 [2] & 6 & DM & $\mathrm{R}$ & No & EM & $\mathrm{S}, \mathrm{E}$ & - \\
\hline CADEN-7, 2007 [26] & 7 & FM & $\mathrm{PA}, \mathrm{R}, \mathrm{T}, \mathrm{H}$ & No & BDCM & $\mathrm{S}, \mathrm{E}, \mathrm{F}, \mathrm{W}$ & PID, EMG \\
\hline IntelliArm, 2009 [39] & 7 & FM & $\mathrm{R}, \mathrm{MS}$ & No & EM & S, E, F, W & VRC \\
\hline L-EXOS, 2009 [10] & 5 & $\mathrm{FM}$ & $\mathrm{R}$ & Yes & DCSM & $\mathrm{S}, \mathrm{E}, \mathrm{F}$ & IMC \\
\hline MGA, 2009 [5] & 6 & $\mathrm{FM}$ & $\mathrm{R}$ & No & BLDCM & S, E, W & $\mathrm{ADC}, \mathrm{IMC}$ \\
\hline RUPERT, 2007 [36] & 4 & $\mathrm{BM}$ & $\mathrm{R}$ & Yes & PMA & S, E, W & FFC \\
\hline ARMin-III, 2009 [25] & 4 & FM & $\mathrm{R}$ & No & BDCM & S, E & PD, CTC, IMC \\
\hline SUEFUL-7, 2009 [12] & 7 & $\mathrm{CM}$ & PA & No & DCSM & $\mathrm{S}, \mathrm{E}, \mathrm{F}, \mathrm{W}$ & FC, EMG \\
\hline SARCOS master arm [24] & 7 & FM & MS & No & HA & S, E, F, W & $\mathrm{PD}, \mathrm{CTC}$ \\
\hline
\end{tabular}

Type-2 End-effector type: These are the kind of devices which do not actively support or hold the subject's upper-limb but connect with the subject's hand or forearm

\begin{tabular}{|c|c|c|}
\hline Name/year & Arm support & Actuation/operating mechanism/brief description \\
\hline MIT-MANUS, 2000 [21] & Forearm, Wrist & $\begin{array}{l}\text { The 1st version of this device used a 3DOFs planer robot to provide } \\
\text { physical therapy of stroke victims. A later version of which [26] includes } \\
\text { hand module for whole arm rehabilitation }\end{array}$ \\
\hline iPAM system, [8] & Upper arm, Wrist & $\begin{array}{l}\text { This system uses a dual robotic arm (each having } 3 \text { active DoFs) to deliver } \\
\text { therapy via two orthoses located on the upper arm and wrist of the } \\
\text { subjects }\end{array}$ \\
\hline HWARD, 2008 [37] & Wrist & $\begin{array}{l}\text { This system is a 3DOFs desk-mounted pneumatically actuated device that } \\
\text { was developed to assist the subject's hand in grasp and in release } \\
\text { movements }\end{array}$ \\
\hline MIME system, 2000 [4] & Forearm & $\begin{array}{l}\text { The system incorporated a PUMA- } 260 \text { robot and two commercial mobile } \\
\text { arm supports modified to limit arm movement to the horizontal plane } \\
\text { (2D); a later version uses PUMA-560 to provide therapy in 3D } \\
\text { workspace }\end{array}$ \\
\hline Homma \& Arai, 1995 [17] & Forearm, wrist & $\begin{array}{l}\text { The system used a parallel mechanism to suspend the upper arm at the } \\
\text { elbow and wrist level }\end{array}$ \\
\hline GENTLE/s system, 2003 [22] & Through Wrist Orthosis & $\begin{array}{l}\text { The system utilizes an active 3DOFs haptic master robot that connects the } \\
\text { subject's arm through a wrist orthosis and uses virtual reality (VR) } \\
\text { technologies to deliver therapy subject's arm through a wrist orthosis and } \\
\text { uses virtual reality (VR) technologies to deliver therapy }\end{array}$ \\
\hline
\end{tabular}

Placement: FM - Floor mounted; DM - Desk mounted CM - Chair mounted. Purpose: R - Rehabilitation; MA - Motion assist; PA - Power assist; MS - Movement study; T - Teleportation; H - Haptci device. Actuators: BLDCM - Brushless DC motor; BDCM - Brushed DC motor; DCSM DC servo motor; EM - Electric motors; PMA - Pneumatic muscle actuator; HA - Hydraulic actuators. Motion Support: S - Shoulder, E - Elbow, F, Forearm, W - wrist, H - Hand (finger). Control: FF - Force feedback; FFC - Feedforward control; FC - Force Control; PID - Proportional integral derivative; PD - Proportional derivative; VRC - Virtual reality based control; CTC - Computed torque control; SMC - Sliding mode control; EMG - Electromyogram based control; IMC - Impedance control; ADC - Admittance control.

joint movement [29] considering elbow joint motion is widely used in performing all daily activities. In a continuing effort toward to make a complete (i.e., 7DoFs) upper-arm motion assisted robotic exoskeleton in this paper we have focused on the development of 5DoFs $M A R S E-5$ to assist elbow, forearm and shoulder joint movements.

Although extensive research has been carried out $[10,12,13,17,25,26,32]$ through last decades and much progress has been made, we are still far behind to develop intelligent, autonomous robots to perform smart tasks such as providing passive/active rehabilitation therapy as well as to provide movement assistance for daily upper-limb motion. A brief description of the state-of-the art with respect to notable rehabilitating devices or robots is presented in Table 1.

Our survey of the recent literature revealed some of the limitations of existing exoskeleton systems, 
which encouraged and motivated us to go through this research. In particular, exoskeleton systems have been designed with limited degrees of freedom and range of motion compared to that of human upper extremities $[10,11,17,37]$. Others have employed a robust and complex structure [39], are relatively heavy, with bulky joints [5], or have a relatively weak joint mechanisms $[17,19]$. Some show a lack of proper safety measures and compensation for gravity forces $[8,17,37]$. Some have been designed using a closed circular structure of arm holder $[12,13]$, making it unrealistic and inconvenient to insert and remove the arm. The use of wire ropes or complex cable routing as a transmission mechanism has been an approach in other types of robots $[10,19,26]$, which can produce undesirable vibration and excessive compliance in the system. Problems can become severe when transmission wire ropes and/or cable slide away from the guide pulleys.

Apart from the hardware limitations, developing intelligent control algorithms is another major issue that needs to be properly addressed to satisfy the desire of its users.

While designing the MARSE-5, we have considered the above limitations; it is fabricated with aluminum to provide the exoskeleton with a relatively light weight structure compared to existing exoskeletons and powered assist devices; it can be easily fitted or removed; has an extended range of movement; and is able to effectively compensate for gravity. To avoid complex cable routing that could be found in many exoskeleton systems $[10,19,26]$, a novel power transmission mechanism has been introduced for assisting shoulder joint internal/external rotation and for forearm pronation/supination. Note that, cable transmission always adds some undesirable vibration and excessive compliance and can loosen up during operation, therefore it should be avoided. On the other hand, it is practically impossible to use conventional gear mechanisms, since in such a case, meshing gears are supposed to rotate around a physical axis of rotation (e.g., shaft), but we are unable to fit such a mechanical shaft along the line of axis of human arm motion (e.g., with the humerus/radius) especially in case of shoulder joint internal/external rotation and for forearm pronation/ supination. To solve these problems, we have introduced an innovative concept of power transmission, a combination of novel custom-made open-type bearing and open-type meshing gear assembly, where motion is transmitted from an anti-backlash gear (mounted on a motor shaft) to an open-type custom-made meshing ring gear. A detail of this mechanism is discussed in Section 3.

The exoskeleton is supposed to be worn on the lateral side of the upper arm and will assist shoulder joint for horizontal and vertical flexion/ extension motion, and internal/external rotation; elbow joint for flexion/extension motion, and forearm for pronation/supination movements. Modified Denavit Hartenberg (DH) conventions [15] were used in developing the kinematic model. In dynamic modeling and control, robot parameters such as MARSE-5 arm link lengths, upper-limb masses and inertia, are estimated according to the upper limb properties of a typical adult [14, 31]. Considering the dynamic of upper-limb movement which is found to be nonlinear in nature, we have applied a nonlinear SMC [35] technique, in trajectories tracking (corresponding to typical rehabilitation exercises [1]) of the developed exoskeleton. The robustness of the SMC can theoretically ensure perfect tracking performance despite parameters or model uncertainties [35]. We therefore considered the SMC as a good potential solution to deliver a consistently high dynamic tracking performance. Experiments were conducted to evaluate the performance of the MARSE-5 to follow the desired trajectories. The trial results revealed that the MARSE-5 is able to track the desired trajectories very efficiently which in this case consisted in passive arm movements, representing passive rehabilitation therapy [1].

In the next section of this paper, the kinematic model for the proposed MARSE-5 is presented. A detail on the design and development of the exoskeleton is presented in Section 3. Section 4 describes its control strategy (SMC). In Section 5, experimental results are presented to evaluate the performance of the MARSE-5 regard to trajectory tracking which corresponds to passive arm movements and finally the paper ends with the conclusion and future works in Section 6.

\section{Kinematic model}

To provide natuaralistics upper-limb movements, the proposed 5DoFs MARSE-5 was modeled based on human upper limb biomechanics. Considering the safety of the robot users and to assist in performing essential daily activities e.g., eating, reaching, grasping, washing the body etc., preliminary studies on the anatomical range $[14,31]$ of upper limb movements have been done to choose a suitable movable range for 


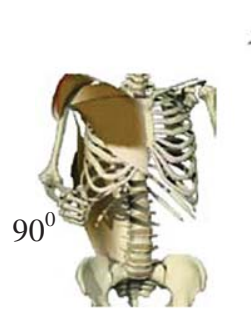

(a)

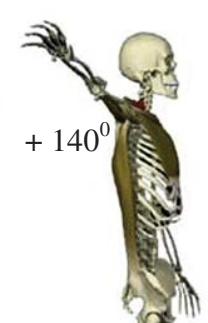

(b)

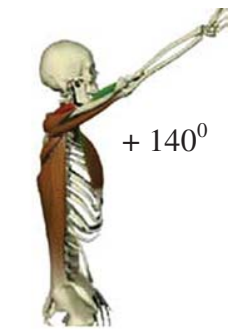

(c)

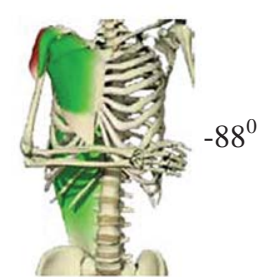

(d)

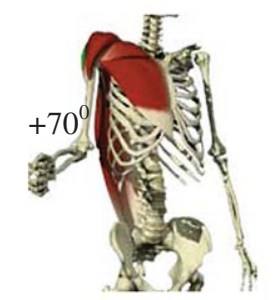

(e)

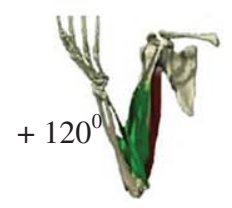

(f)

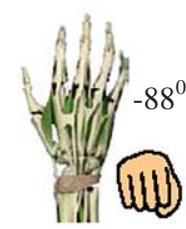

(g)

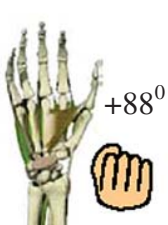

(h)
Fig. 1. Workspace of MARSE-5. (a) Initial position; (b) Shoulder joint: abduction, (c) Shoulder joint: vertical flexion; (d) Shoulder joint: internal rotation; (e) Shoulder joint: external rotation; (f) Elbow flexion; (g) Forearm pronation; (h) Forearm supination.

the MARSE-5. The movable range of the MARSE-5 is depicted in Fig. 1.

To develop the kinematic model of the MARSE-5, the link-frame attachments are depicted in Fig. 2. The joint axes of rotation of human upper limb corresponding to the MARSE-5 are indicated by dark black arrow heads (i.e., $Z$-axes). In this model, joints 1,2 , and 3 together constitute the glenohumeral joint, commonly known as the shoulder joint, where joint 1 corresponds to horizontal flexion/extension, joint 2 to vertical flexion/extension, and joint 3 to internal/external rotation. Note that for this exoskeleton robot, the axes of joints 1,2 , and 3 (i.e., $Z_{1-3}$ ) all intersect at a common point (point-B, Fig. 3). Joint 4, which is located at a distance $d_{h}$ (length of humerus) apart from the shoulder joint, corresponds to flexion/extension of the elbow joint. As also seen in Fig. 2, joint 5, representing the pronation/supination of the forearm coincides with joint 4 and is located at a distance (radial/ulnar) $d_{w}$ from wrist joint.

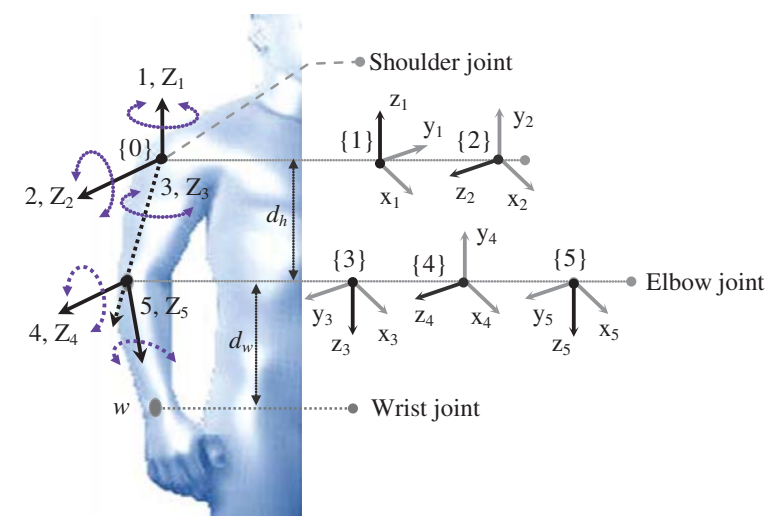

Fig. 2. Link frame attachments.

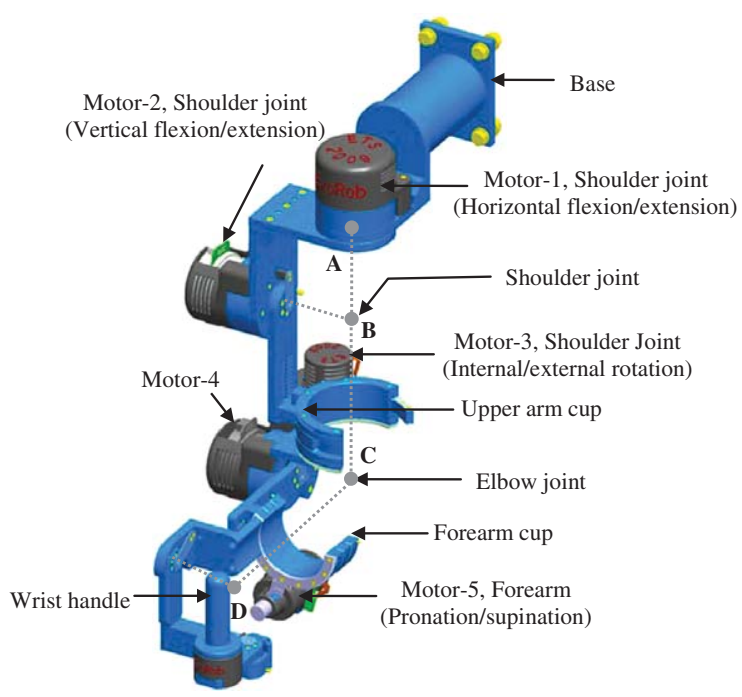

Fig. 3. A 5 DoFs MARSE arm, (Right hand side view).

To get the DH parameters we assume that the coordinate frames (i.e., the link-frames which map between the successive axes of rotation) coincide with the joint axes of rotation and have the same number of order, i.e., frame $\{1\}$ coincides with joint 1 , and frame $\{2\}$ with joint 2, and so on. The modified DH parameters corresponding to the placement of link frames (in Fig. 2) are summarized in Table 2. These DH parameters are used to get homogeneous transfer matrix [7] which represents the positions and orientations of the reference frame with respect to the fixed reference frame. It is considered that the fixed reference frame $\{0\}$ coincides with the first reference frame. 
Table 2

Modified denavit-hartenberg parameters

\begin{tabular}{lcccc}
\hline Joint (i) & $a_{i-1}$ & $a_{i-1}$ & $d_{i}$ & $\theta_{i}$ \\
\hline 1 & 0 & 0 & 0 & $\theta_{1}$ \\
2 & $\pi / 2$ & 0 & 0 & $\theta_{2}$ \\
3 & $\pi / 2$ & 0 & $\mathrm{~d}_{h}$ & $\theta_{3}$ \\
4 & $-\pi / 2$ & 0 & 0 & $\theta_{4}$ \\
5 & $\pi / 2$ & 0 & 0 & $\theta_{5}$ \\
$w$ & 0 & 0 & $\mathrm{~d}_{w}$ & 0 \\
\hline
\end{tabular}

where, $a_{i-1}$ is the link twist; $a_{i-1}$ corresponds to link length; $d_{i}$ is the link offset; and $\theta_{i}$ is the joint angle.

We know that the general form of a link transformation that relates frame $\{i\}$ relative to the frame $\{i-1\}$ [7] is-

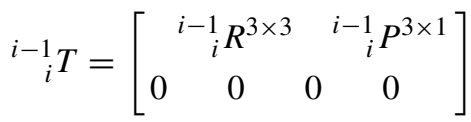

where, ${ }^{i-1} R$ is the rotation matrix that describes frame $\{i\}$ relative to frame $\{i-1\}$ and can be expressed as:

$$
\begin{aligned}
& { }_{i}^{i-1} R \\
& =\left[\begin{array}{ccc}
\cos \theta_{i} & -\sin \theta_{i} & 0 \\
\sin \theta_{i} \cos \alpha_{i-1} & \cos \theta_{i} \cos \alpha_{i-1} & -\sin \alpha_{i-1} \\
\sin \theta_{i} \sin \alpha_{i-1} & \cos \theta_{i} \sin \alpha_{i-1} & \cos \alpha_{i-1}
\end{array}\right] \text { (2) }
\end{aligned}
$$

and, ${ }_{i}^{i-1} P$ is the vector that locates the origin of frame $\{i\}$ relative to frame $\{i-1\}$ and can be expressed as:

$$
{ }_{i}^{i-1} P=\left[a_{i-1}-s a_{i-1} d_{i} c a_{i-1} d_{i}\right]^{T}
$$

Using Equation (1-3), the individual homogeneous transfer matrix that relates two successive frame (of Fig. 2) can be found as:

$$
\begin{aligned}
{ }_{1}^{0} T= & {\left[\begin{array}{cccc}
\cos \theta_{1} & -\sin \theta_{1} & 0 & 0 \\
\sin \theta_{1} & \cos \theta_{1} & 0 & 0 \\
0 & 0 & 1 & 0 \\
0 & 0 & 0 & 1
\end{array}\right], } \\
{ }_{2}^{1} T= & {\left[\begin{array}{cccc}
\cos \theta_{2} & -\sin \theta_{2} & 0 & 0 \\
0 & 0 & -1 & 0 \\
\sin \theta_{2} & \cos \theta_{2} & 0 & 0 \\
0 & 0 & 0 & 1
\end{array}\right] }
\end{aligned}
$$

$$
\begin{aligned}
{ }_{3}^{2} T= & {\left[\begin{array}{cccc}
\cos \theta_{3} & -\sin \theta_{3} & 0 & 0 \\
0 & 0 & -1 & -d_{h} \\
\sin \theta_{3} & \cos \theta_{3} & 0 & 0 \\
0 & 0 & 0 & 1
\end{array}\right], } \\
{ }_{4}^{3} T= & {\left[\begin{array}{cccc}
\cos \theta_{4} & -\sin \theta_{4} & 0 & 0 \\
0 & 0 & 1 & 0 \\
-\sin \theta_{4} & -\cos \theta_{4} & 0 & 0 \\
0 & 0 & 0 & 1
\end{array}\right] } \\
{ }_{5}^{4} T= & {\left[\begin{array}{cccc}
\cos \theta_{5} & -\sin \theta_{5} & 0 & 0 \\
0 & 0 & -1 & 0 \\
\sin \theta_{5} & \cos \theta_{5} & 0 & 0 \\
0 & 0 & 0 & 1
\end{array}\right], } \\
{ }_{w}^{5} T= & {\left[\begin{array}{cccc}
1 & 0 & 0 & 0 \\
0 & 1 & 0 & 0 \\
0 & 0 & 1 & d_{w} \\
0 & 0 & 0 & 1
\end{array}\right] . }
\end{aligned}
$$

The homogenous transformation matrix that relates frame $\{w\}$ to frame $\{0\}$ can be obtained by multiplying individual transformation matrices.

$$
{ }_{w}^{0} T={ }_{1}^{0} T \cdot{ }_{2}^{1} T \cdot{ }_{3}^{2} T{ }_{4}^{3} T \cdot{ }_{5}^{4} T \cdot{ }_{w}^{5} T
$$

The single transformation matrix thus found from Equation (5) represents the positions and orientations of the reference frame attached to the wrist joint (joint$w$ ) with respect to the fixed reference frame $\{0\}$.

\section{Motion Assistive Robotic-exoskeleton for Superior Extremity (MARSE-5)}

The 5DoFs MARSE-5, as shown in Fig. 3, is comprised of a shoulder motion support part, an elbow motion support part and a forearm motion support part. The entire MARSE-5 arm is fabricated with aluminum to provide the exoskeleton structure with a relatively light weight, considering aluminum is a low density material having reasonable strength characteristics. The mass and inertia properties of the MARSE-5 are summarized in Table 3. 
Table 3

Mass and inertia properties of MARSE-5*

\begin{tabular}{|c|c|c|c|c|c|c|c|c|}
\hline \multirow{2}{*}{$\begin{array}{l}\text { Segment } \\
\text { (Fig. 3) }\end{array}$} & \multirow{2}{*}{$\begin{array}{c}\text { Segment } \\
\text { length }(\mathrm{cm})\end{array}$} & \multirow{2}{*}{$\begin{array}{c}\text { Segment } \\
\text { weight }(\mathrm{kg})\end{array}$} & \multicolumn{3}{|c|}{ Centre of gravity $C G(\mathrm{~cm})$} & \multicolumn{3}{|c|}{ Moment of inertia $I\left(\mathrm{~kg} \cdot \mathrm{m}^{2}\right)$} \\
\hline & & & $C G_{X}$ & $C G_{Y}$ & $C G_{Z}$ & $I x x$ & Iyy & $I z z$ \\
\hline Shoulder joint ${ }^{\mathrm{A}}$ & 14.0 & 3.47 & 0.007 & -13.82 & -9.84 & 0.0232 & 0.0148 & 0.013 \\
\hline Upper $\operatorname{arm}^{\mathrm{B}}$ & $25 \pm 8.85$ & 3.737 & -1.31 & -9.79 & 19.5 & 0.0233 & 0.0128 & 0.020 \\
\hline Forearm ${ }^{\mathrm{C}}$ & $26 \pm 4.75$ & 2.066 & -2.93 & -16.32 & 5.89 & 0.0166 & 0.0100 & 0.0126 \\
\hline
\end{tabular}

${ }^{A}$ Point-A to point-B (shoulder joint, Fig. 3); ${ }^{B}$ Shoulder joint to Elbow (point-C, Fig. 3); ${ }^{C}$ Elbow to Wrist (point-D, Fig. 3); *The mass and inertia properties of the MARSE-5 were estimated from the CAD modelling using Pro/Engineer software.

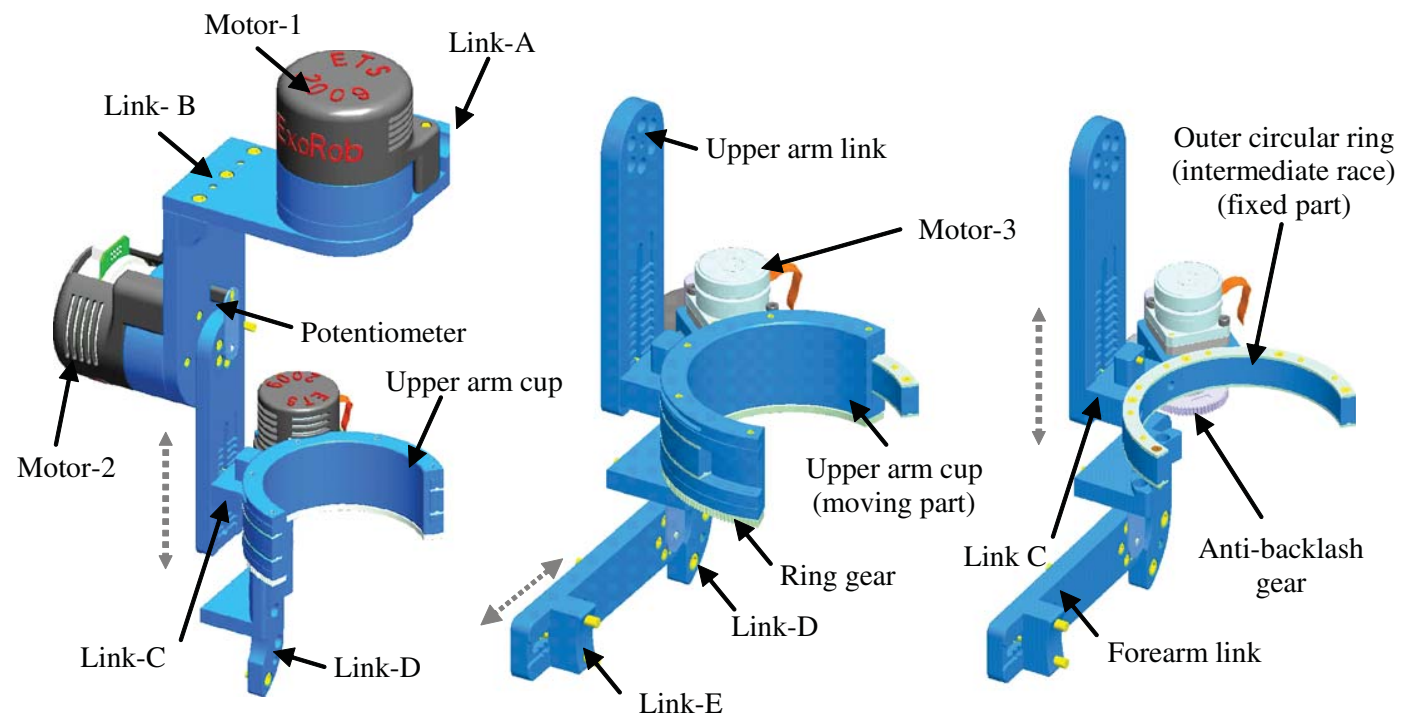

(a)

(b)

(c)

Fig. 4. (a) Shoulder motion support part (horizontal and vertical flexion/extension); (b) Shoulder joint internal/external rotation support part (when elbow motor is unplugged from elbow joint). Figure shows upper arm cup rotation over the custom made bearing; (c) Intermediate race assembly with the upper arm link.

\subsection{Shoulder motion support part (horizontal and vertical flexion/extension)}

The shoulder joint motion support part has 3DoFs and is able to assist with horizontal and vertical flexion/extension motion, and internal/external rotation of shoulder joint. It consists of two motors (Maxon EC-90), two links (link-A, and link-B), and two potentiometers. The link-A holds the motor- 1 at its one end (Fig. 4) and is rigidly fixed to the base structure of the robot (Fig. 3) at its other end. As shown in Fig. 4, the link-B, which is hinged with the motor- 1 and carries the motor- 2 on its other end, is ' $\mathrm{L}$ ' shaped, in order to accommodate the subject's shoulder joint. Therefore, the axes of rotation of motors 1 and 2 are supposed to intersect at the centre of rotation of subject's shoulder joint (point-B, Fig. 3). Moreover, by adjusting the seating height (e.g., using a height adjustable chair) it would be easy to align the centre of rotation of the shoulder joint of subject to that of the MARSE-5. Note that motor-1 is responsible for shoulder joint horizontal flexion/extension motion and motor-2 is for vertical flexion/extension motion. It is worth mentioning here that there is no scapular elevation but rather rotation during the abduction of the GHJ [16]. However, the scapular elevation of subjects which is common due to GHJ flexion will be allowed normally during the vertical flexion motion of the developed MARSE-5 and there should be no discomfort to the subject if the centre of rotation of shoulder joint of the subject aligned with that of MARSE-5. 


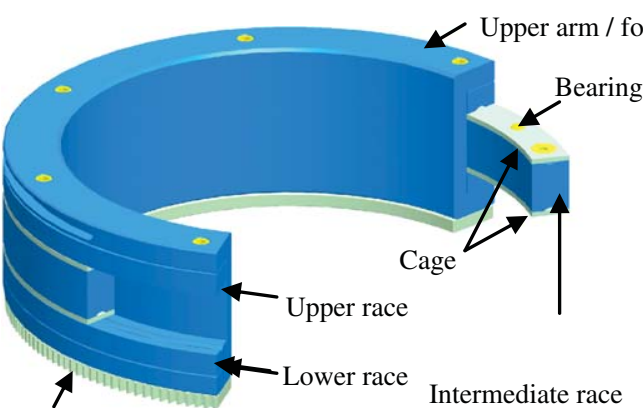

Ring gear

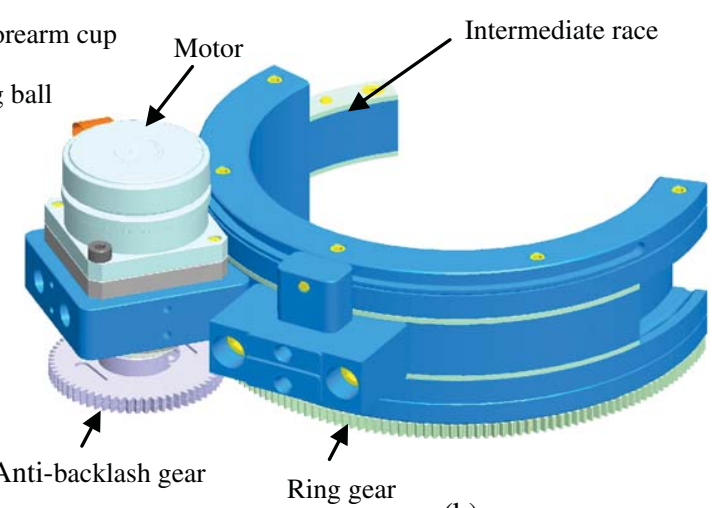

(b)

Fig. 5. (a) An open type bearing assembly, (b) Actuation mechanism with an open type bearing and a ring gear.

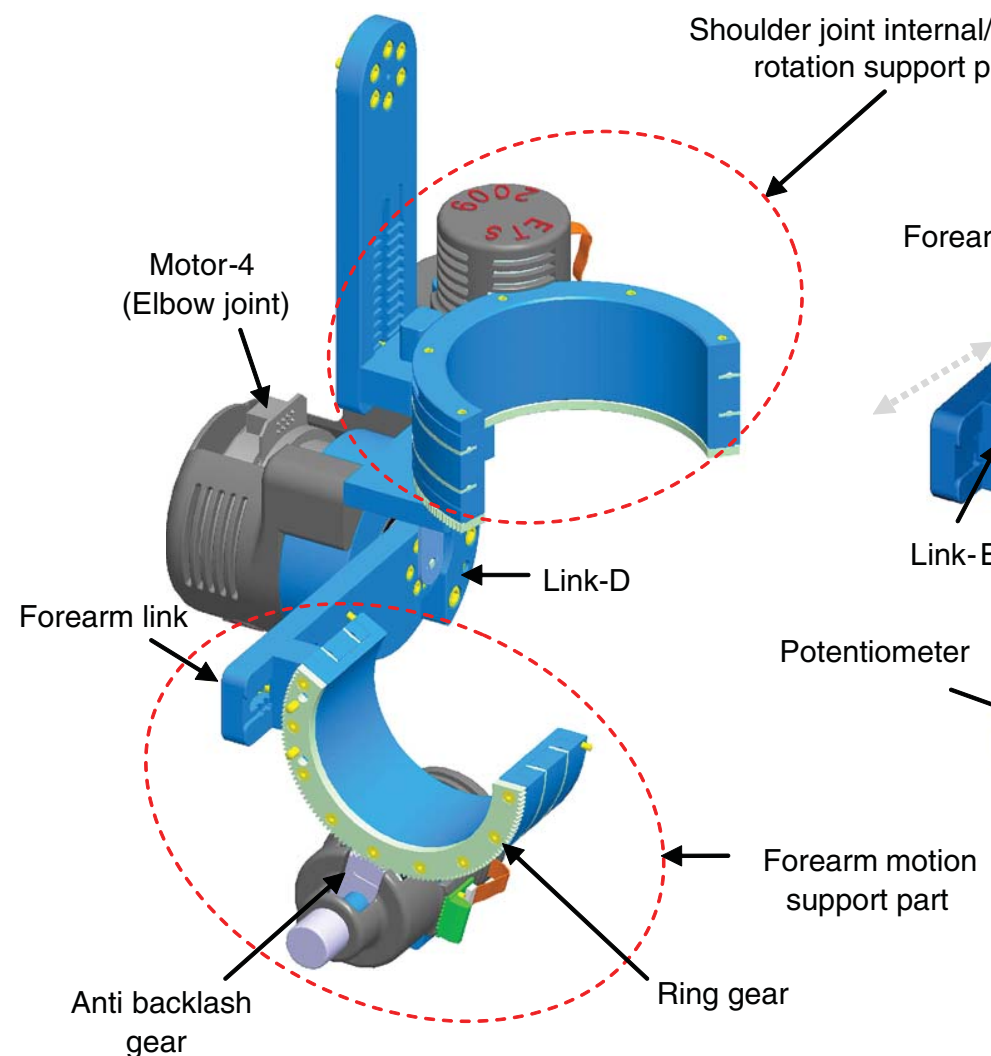

(a) (b)

Fig. 6. (a) Elbow and forearm motion support part, (b) Forearm motion support part (when forearm cup is not assembled).

The actuation mechanisms developed for the shoulder joint internal/external rotation support part and the forearm motion support part are somewhat complex, as it is impossible to place any actuator along the axis of rotation of the upper arm (e.g., with the humerus/radius), due to the anatomical configuration of the human arm. Some devices used gear mechanism with a closed circular structure of forearm/upper arm 
cup $[12,13]$. However it is unrealistic and inconvenient to insert and remove the arm through a closed circular structure. Other devices make use of a complex cable transmission mechanism to assist with forearm motion $[10,26]$. One of the major limitations of such cable driven systems is that it delivers undesirable vibration and excessive compliance to the system. To deal with this problem, this research introduced an innovative concept of power transmission, a combination of a custom-made open-type bearing and open type meshing gear assembly, where motion is transmitted from an anti-backlash gear (mounted on a motor shaft) to an open type, custom-made meshing ring gear that is rigidly attached to the open type upper/forearm cup. A detail of this transmission mechanism is discussed below.

\subsection{A new power transmission mechanism (Alternate gear mechanism)}

The transmission mechanism as proposed in this research introduced the concept and the development of an open type bearing. Unlike conventional bearing, this open type bearing make use of two layers of bearing balls (Fig. 5), therefore it required two specially designed bearing ball cages. Moreover it has three bearing races (upper race, intermediate race, and lower race, Fig. 5) instead of two as often found in the conventional type of bearing. Figure 5 shows the intermediate race which is designed to hold stainless steel balls ( $4 \mathrm{~mm}$ diameters) on its two sides by using the bearing balls cages. The upper and lower races assembly is shown in Fig. 5a, where it can be found that the bearing races were assembled with the upper arm or forearm cup. As depicted also in Fig. 5a, the ring gear, which is supposed to be used in transmitting power from the actuator, is assembled underneath the arm cup. Note that the ball bearings are positioned between the groove of the intermediate race and the upper/lower races, and act as a frictionless rotating mechanism. The proposed actuation mechanism which, is a combination of open type gear and bearing, is depicted in Fig. 5b, where it can be seen that the actuator (motor) is rigidly mounted on the back of the intermediate race. It is the anti-backlash gear which is clamped along the motor shaft transmits the actuator (rotary) motion to the ring gear. Since the ring gear is firmly fixed to the arm cup, it rotates the arm cup as well over the custom-designed open type bearing.

\subsection{Shoulder motion support part (internal/external rotation)}

To assist with shoulder joint internal/external rotation, the MARSE-5 is comprised of an upper arm link, a sliding link (link-C), a motor (Maxon EC-45), a potentiometer, and an alternate gear mechanism as discussed above (i.e., a custom-made open type bearing, a ring gear, and an anti-backlash gear assembly). The upper-arm link, as shown in Fig. 4b, is hinged with the motor-2 (Fig. 4) and holds the entire MARSE arm. The link-C (Fig. 4c) is rigidly fixed with the outer circular ring (i.e., with the intermediate race of the bearing) and is able to slide along the upper arm link (Fig. 4c, dotted arrow) so that the distance between the upper arm cup and shoulder joint (as well as the distance between elbow joint and shoulder joint) may be adjusted to accommodate a wide range of users. Note that a detail study on the biomechanics of upper extremity was performed (within the age group 20-65) [14, 31, 40] in designing the MARSE-5, therefore provision includes in the design to adjust the link-length for the convenience of the user, which is one of the key aspect of the design. The open half-circular structure of the upper arm cup allows users to position the arm easily, without having to insert the arm through a closed circular structure. As depicted in Figs. $4 \mathrm{~b}$ and c, the motor-3 (Maxon EC-45) is rigidly mounted on the back of the intermediate race (i.e., with the fixed outer ring). Figure $4 \mathrm{c}$ shows the anti-backlash gear which is clamped along the motor shaft to transmit the rotary motion to the ring gear. As discussed previously in the development of alternate gear mechanism, since the ring gear is firmly fixed underneath the upper arm cup (Fig. 4b), it is therefore responsible for rotation of the upper arm cup over the custom-designed open type bearing.

\subsection{Elbow and forearm motion support part}

The elbow motion support part is comprised of a forearm link, a fixed link (Link-D), a motor (Maxon EC-90) and a potentiometer. As shown in Fig. 6, the link-D acts as a bridge between the shoulder joint internal/external rotation support part and the elbow motion support part. Its one end is assembled with the upperarm cup and with the other end it holds the elbow motor as well as the elbow motion support part. The forearm link as depicted in Fig. 6a is hinged with the elbow motor (motor-4) at the elbow joint (Fig. 3) and carry the entire forearm motion support part. 


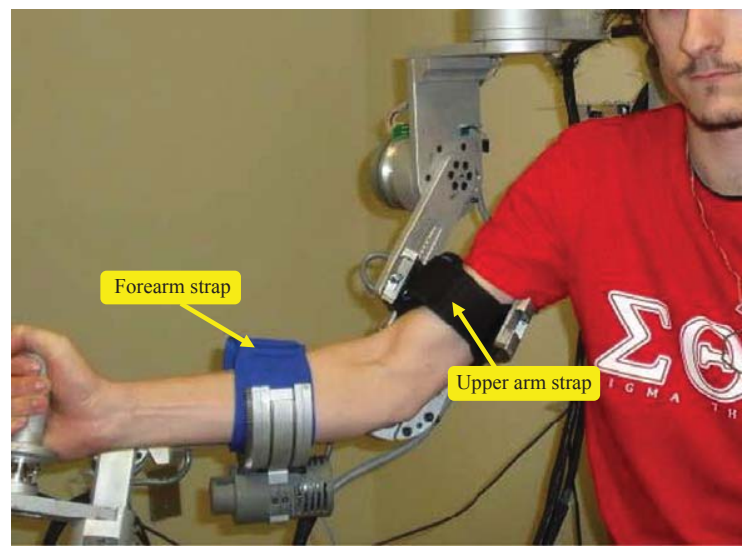

Fig. 7. MARSE-5 with its user.

The forearm motion support part consists of a sliding link (link-E), a motor (Maxon EC-45), a potentiometer, and an alternate gear mechanism (i.e., a custom made open type bearing, a ring gear, and an anti-backlash gear assembly). The sliding link (link-E) is rigidly fixed with the intermediate race (i.e., outer circular ring as depicted in Fig. 6b) and is able to slide along the forearm link (Fig. 6b dotted arrow) to adjust the distance between the forearm strap and the elbow joint (as well as to adjust the distance between the elbow and wrist joints). The design principle of the forearm motion support part is quite similar to that of the shoulder joint internal/external support part. As for the upper-arm cup, the open half circular structure of the forearm cup allows users to place and position their forearm easily, without having to insert the forearm through a closed circular structure. The motor (Maxon-EC45) is rigidly mounted on the back of the fixed outer circular ring. Figure $6 a$ shows the anti-backlash gear, which is clamped along the motor shaft to transmit the rotary motion to the ring gear. As also shown in Fig. 6a the ring gear (open type) is firmly fixed to the forearm arm cup and is responsible to rotate the forearm arm cup over the custom-designed open type bearing. Note that to hold the upper-arm/forearm in a proper position, soft arm straps (Fig. 7) are pasted on the upper-arm and forearm cups. The movable range of the MARSE-5 is summarized in Table 4, as compared to motion of the human upper-limb.

Note that the actuators used for the MARSE-5 are brushless DC motors. Harmonic drives (HD) are incorporated into the motors in order to increase the torque and to reduce the speed of rotation. Detail specifications of actuators are given in Appendix-A.
Table 4

Range of movement

\begin{tabular}{lcccc}
\hline Types of motion & \multicolumn{3}{c}{ Anatomical range [14] } & $\begin{array}{c}\text { MARSE-5's } \\
\text { workspace }\end{array}$ \\
\cline { 2 - 4 } & Source 1 & Source 2 & Source 3 & \\
\hline Shoulder joint & & & & \\
Flexion & $180^{\circ}$ & $170^{\circ}$ & $180^{\circ}$ & $140^{\circ}$ \\
Extension & $50^{\circ}$ & $30^{\circ}$ & $60^{\circ}$ & $0^{\circ}$ \\
Abduction & $180^{\circ}$ & $170^{\circ}$ & $180^{\circ}$ & $140^{\circ}$ \\
Adduction & $50^{\circ}$ & - & - & $0^{\circ}$ \\
Internal rotation & $90^{\circ}$ & $90^{\circ}$ & $90^{\circ}$ & $-88^{\circ}$ \\
External rotation & $90^{\circ}$ & $90^{\circ}$ & $60-90^{\circ}$ & $+70^{\circ}$ \\
Elbow and forearm & & & & \\
Flexion & $140^{\circ}$ & $140^{\circ}$ & $145^{\circ}$ & $120^{\circ}$ \\
Extension & $0^{\circ}$ & $0^{\circ}$ & $5-15^{\circ}$ & $0^{\circ}$ \\
Pronation & $80^{\circ}$ & $90^{\circ}$ & $80^{\circ}$ & $-88^{\circ}$ \\
Supination & $80^{\circ}$ & $85^{\circ}$ & $90^{\circ}$ & $+88^{\circ}$ \\
\hline
\end{tabular}

Selection of appropriate actuators and corresponding HDs (to minimize power/weight ratio) involves extensive analyses on upper limb biomechanics (such as joints' range, speed of movements, acceleration, torque requirements, etc.). Therefore, a detailed study was performed in this research to investigate upper limb speed of movement and torque requirements in performing varieties of daily activities (such as reaching to various targets, head reach to head level, eat with spoon etc.) [27, 30, 34, 38]. However it was found from the studies that patient following a brain injury and/or a spinal cord injury took longer time and showed less smooth movement in completing task compared to normal healthy individual's arm movement [27, 38].

\section{Control}

In this paper, we have applied a robust sliding mode control technique [7] for trajectory tracking of the developed MARSE-5. The dynamic behaviour of the $M A R S E-5$ can be expressed by the well-known rigid body dynamic equation as:

$$
M(\theta) \ddot{\theta}+V(\theta, \dot{\theta})+G(\theta)+F(\theta, \dot{\theta})=\tau
$$

where, $\theta \in \mathbb{R}^{5}$ is the joint variables vector, $\tau$ is the generalized torques vector, $M(\theta) \in \mathbb{R}^{5 \times 5}$ is the inertia matrix, $V(\theta, \dot{\theta}) \in \mathbb{R}^{5}$ is the coriolis/centrifugal vector, $G(\theta) \in \mathbb{R}^{5}$ is the gravity vector, and $F(\theta, \dot{\theta}) \in$ $\mathbb{R}^{5}$ is the friction vector. Note that the friction vector is modeled as a nonlinear coulomb friction, and can be expressed as: 


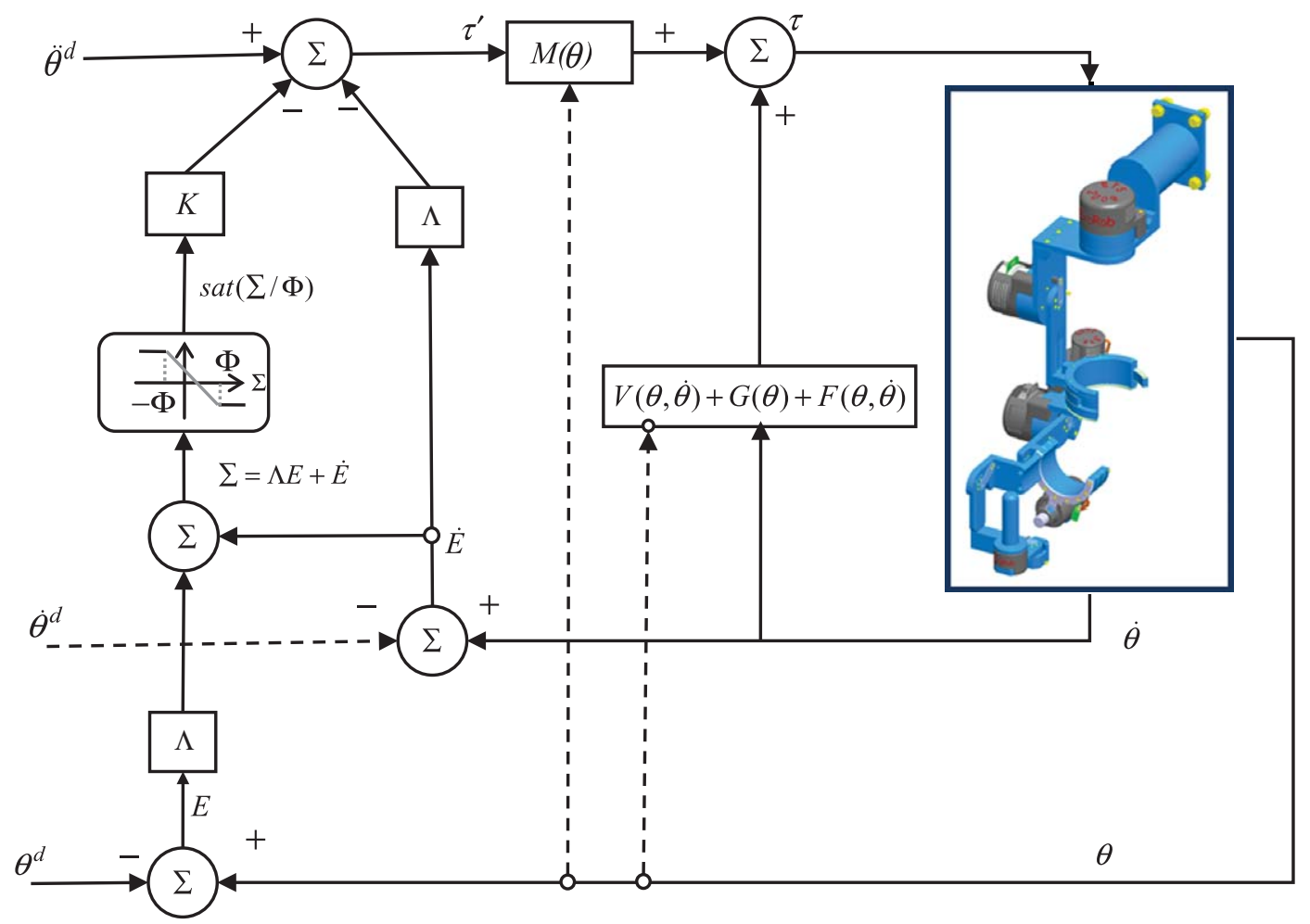

Fig. 8. Schematic diagram of SMC with boundary layer neighbouring to the sliding surface.

$$
\tau_{\text {friction }}=F(\theta, \dot{\theta})=c \cdot \operatorname{sign}(\dot{\theta})
$$

where, $c$ is the coulomb-friction constant. Equation (6) can be written as:

$$
\begin{aligned}
\ddot{\theta}= & -M^{-1}(\theta)[V(\theta, \dot{\theta})+G(\theta)+F(\theta, \dot{\theta})] \\
& +M^{-1}(\theta) \tau
\end{aligned}
$$

$M^{-1}(\theta)$ always exists since $M(\theta)$ is symmetrical and positive definite.

The general layout corresponding to the SMC is depicted in Fig. 8. Note that to eliminate the chattering of conventional SMC, we employed the boundary layer technique [35]. Therefore, we first define the theoretical structure of the conventional SMC, and then modify the algorithm by adding a boundary layer neighboring to the sliding surface.

The first step in the sliding mode control is to choose the sliding (or switching) surface $S$ in terms of the tracking error. Let the tracking error for each joint is defined as:

$$
e_{i}=\theta_{i}-\theta_{i}^{d} \quad \ldots \quad(i=1, \ldots, m)
$$

and the sliding surface as:

$$
S_{i}=\lambda_{i} e_{i}+\dot{e}_{i} \quad \ldots \quad(i=1, \ldots, m)
$$

where, $\theta_{i}^{d}$ is the desired trajectory for joint $i$, and $S_{i}$ is the sliding surface of each DoF.

Let, $\Sigma=\left[\begin{array}{llll}S_{1} & S_{2} \ldots S_{m}\end{array}\right]^{T}$ be the sliding surface for the developed MARSE-5. Therefore, we have,

$$
\Sigma=\left[\begin{array}{c}
\lambda_{1} e_{1}+\dot{e}_{1} \\
\cdot \\
\cdot \\
\cdot \\
\lambda_{m} e_{m}+\dot{e}_{m}
\end{array}\right]
$$

Equation (11) is a first order differential equation, which implies that if the sliding surface is reached, the tracking error will converge to zero as long as the error vector stays on the surface. The convergence rate is in direct relation with the value of $\lambda$. Considering the following Lyapunov function candidate:

$$
V=\frac{1}{2} \Sigma^{T} \Sigma
$$


which is continuous and nonnegative. The derivative of $V$ yields:

$$
\dot{V}=\Sigma^{T} \dot{\Sigma}
$$

By choosing $\dot{\Sigma}$ as given in Equation (14), relation (13) is ensured to be decreasing.

$$
\dot{\Sigma}=-K \cdot \operatorname{sign}(\Sigma), \forall t, K>0 \Rightarrow \dot{V}<0
$$

where,

$$
\operatorname{sign}(\Sigma)=\left\{\begin{array}{c}
1 \text { for } \Sigma>0 \\
0 \text { for } \Sigma=0 \\
-1 \text { for } \Sigma<0
\end{array}\right.
$$

Expression (14) is known as the reaching law for the conventional SMC. It is to be noted that the discontinuous term $K \operatorname{sign}(\Sigma)$ in (14) often leads to a high control activity, known as chattering. The chattering phenomenon is undesirable, because it can excite high frequency dynamics which could be the cause of severe damage. One of the most known approaches found in literature is to smoothen the discontinuous term in the control input with the continuous term $\operatorname{K}$.sat $(\Sigma / \phi)$ [35]. where,

$$
\begin{aligned}
& \operatorname{sat}(\Sigma / \phi) \\
& =\left\{\begin{array}{r}
1 \text { for } \Sigma \geq \phi \\
\frac{\Sigma}{\phi} \text { for }-\phi \leq \Sigma \leq \phi \quad \forall t, 0<\phi \ll 1 \\
-1 \text { for } \Sigma \leq \phi
\end{array}\right.
\end{aligned}
$$

Using Equation (16), the reaching law therefore becomes:

$$
\dot{\Sigma}=-K . s a t(\Sigma / \phi), \forall t, K>0
$$

Therefore and considering:

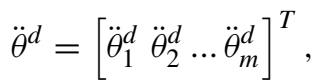

$$
\begin{aligned}
& \dot{E}=\left[\begin{array}{llll}
\dot{e}_{1} & \dot{e}_{2} & \ldots & \dot{e}_{m}
\end{array}\right]^{T} \text {, and } \\
& \wedge=\left[\begin{array}{ccc}
\lambda_{i} & 0 & 0 \\
0 & \ddots & 0 \\
0 & 0 & \lambda_{m}
\end{array}\right] \\
& \Sigma=\Lambda E+\dot{E} \Rightarrow \dot{\Sigma}=\Lambda \dot{E}+\ddot{E}
\end{aligned}
$$

where, $\ddot{E}=\ddot{\theta}-\ddot{\theta}^{d}$. Therefore, relation (18) can be written as:

$$
\dot{\Sigma}=\Lambda \dot{E}+\dot{\theta}-\ddot{\theta}^{d}
$$

Substituting the value of $\ddot{\theta}$ from Equation (8) in Equation (19) we obtain,

$$
\begin{aligned}
\dot{\Sigma}= & \Lambda \dot{E}-\ddot{\theta}^{d}-M^{-1}(\theta) \\
& {[V(\theta, \dot{\theta})+G(\theta)+F(\theta, \dot{\theta})] } \\
+ & M^{-1}(\theta) \tau
\end{aligned}
$$

Replacing $\dot{\Sigma}$ by its value given in Equation (17)

$$
\begin{array}{r}
-K . \operatorname{sat}(\Sigma / \phi)=\Lambda \dot{E}-\ddot{\theta}^{d}-M^{-1}(\theta) \\
{[V(\theta, \dot{\theta})+G(\theta)+F(\theta, \dot{\theta})-\tau]}
\end{array}
$$

The torque $\tau$ can be isolated and thus give:

$$
\begin{aligned}
\tau= & -M(\theta)\left(\Lambda \dot{E}-\ddot{\theta}^{d}+K . \operatorname{sat}(\Sigma / \phi)\right) \\
& +[V(\theta, \dot{\theta})+G(\theta)+F(\theta, \dot{\theta})]
\end{aligned}
$$

Where, $K$ and $\Lambda$ are diagonal positive definite matrices, therefore the control law given in relation (22) ensures that the control system is stable.

\section{Experiments and results}

Experimental set-up for the MARSE-5 system is depicted in Fig. 9. Potentiometers which are incorporated with each joint of the MARSE are sampled at $1 \mathrm{~ms}$.

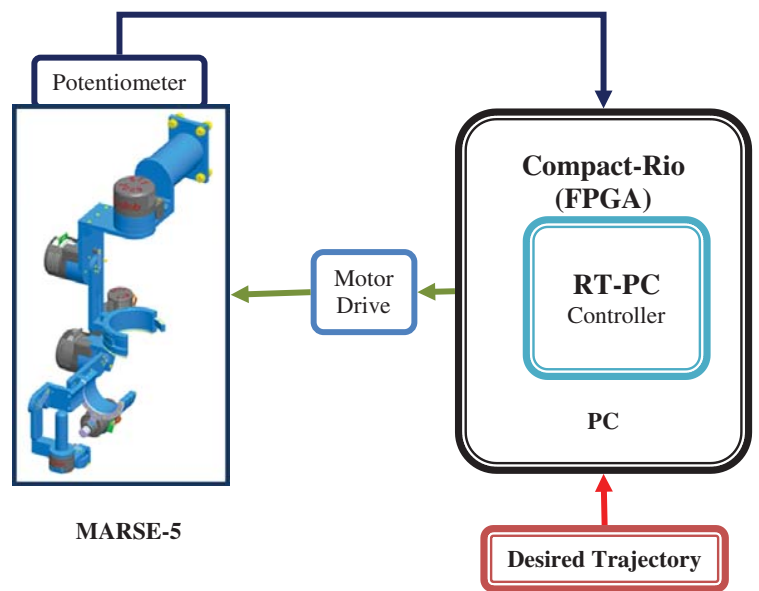

Fig. 9. Experimental setup. 


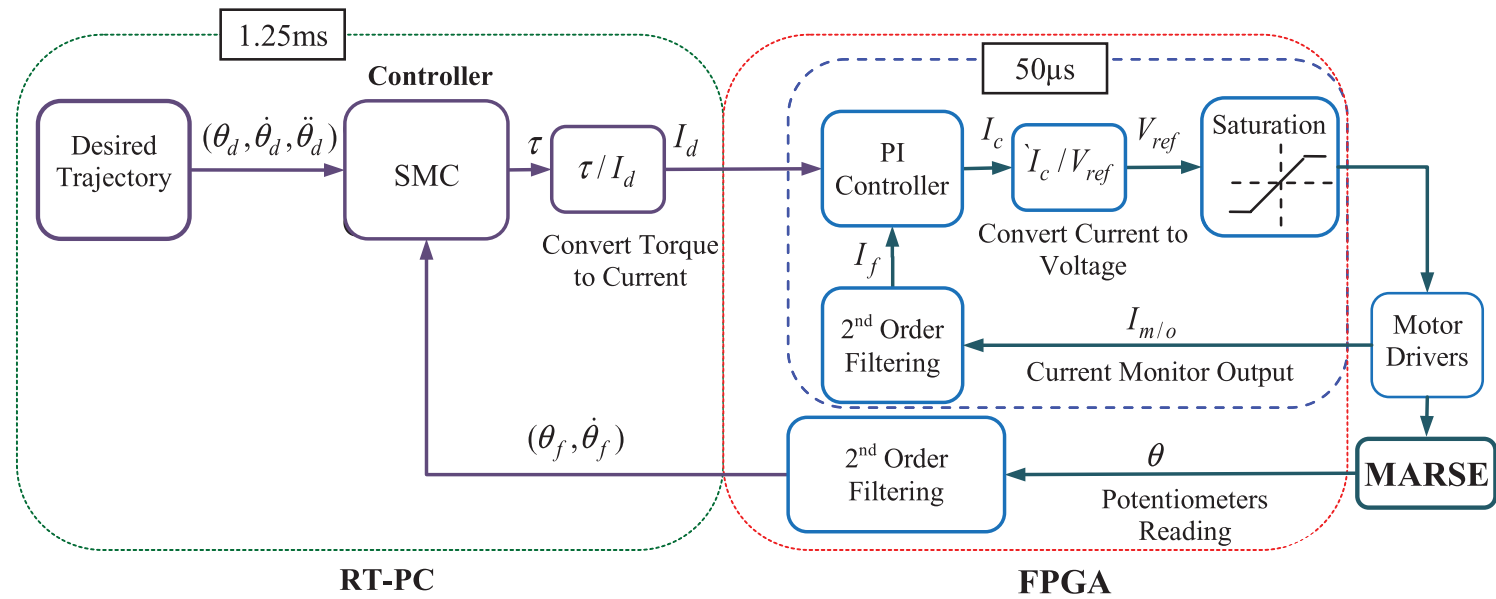

Fig. 10. Control architecture.
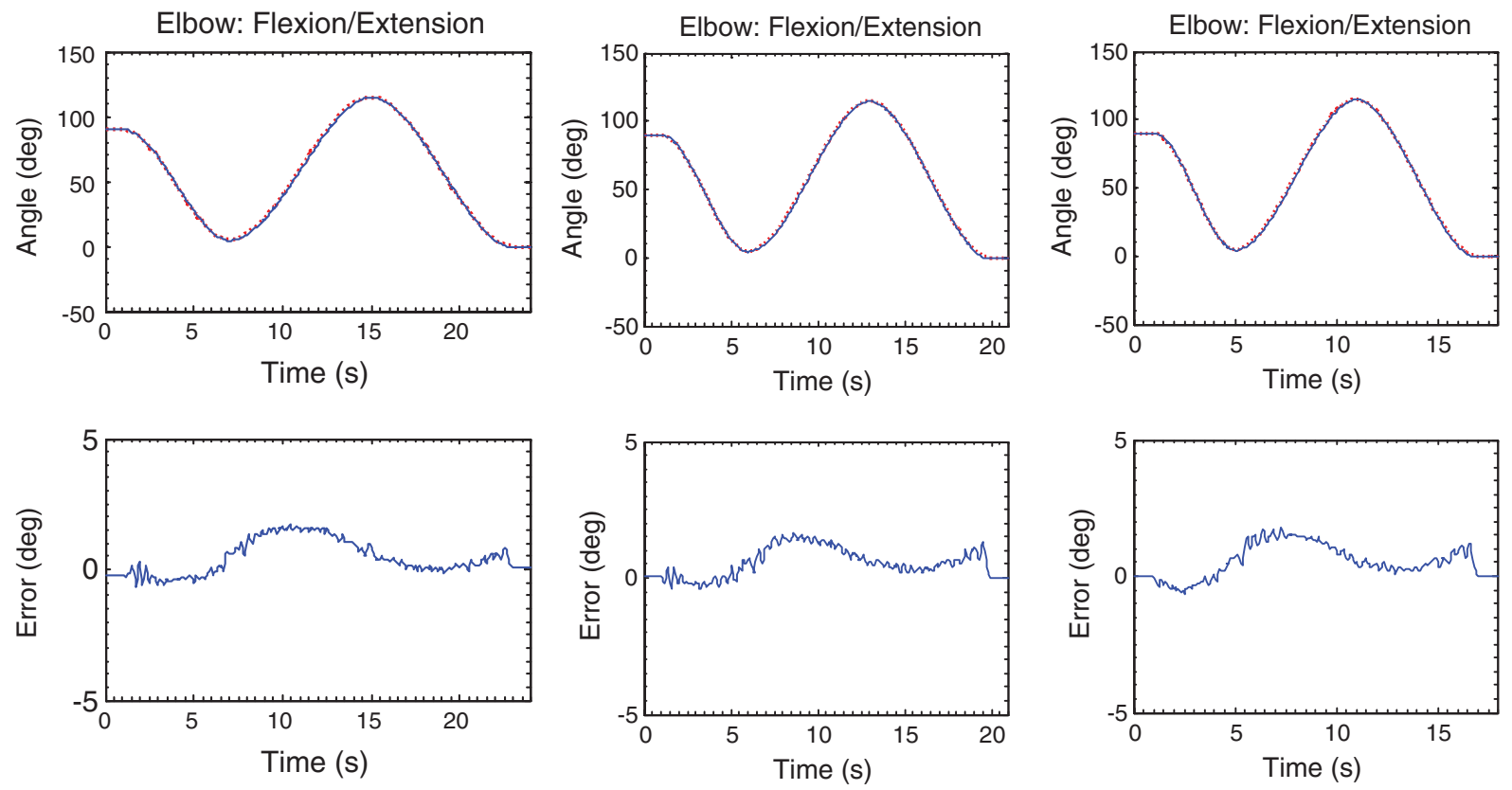

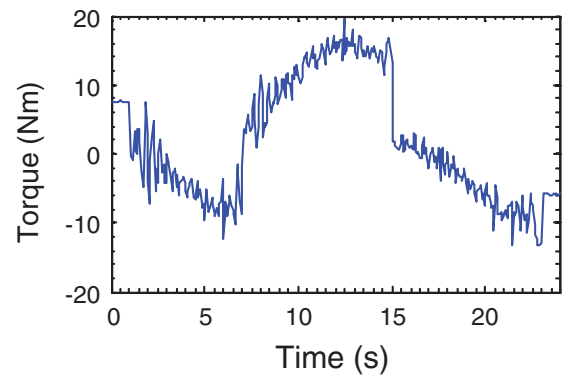

(a)

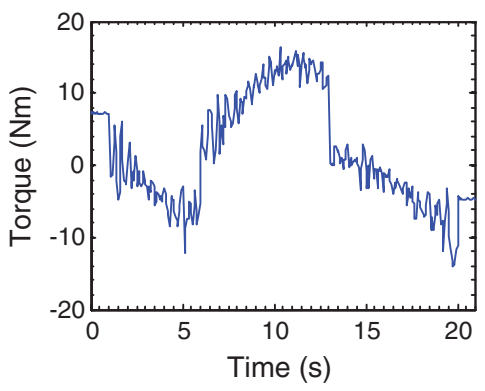

(b)

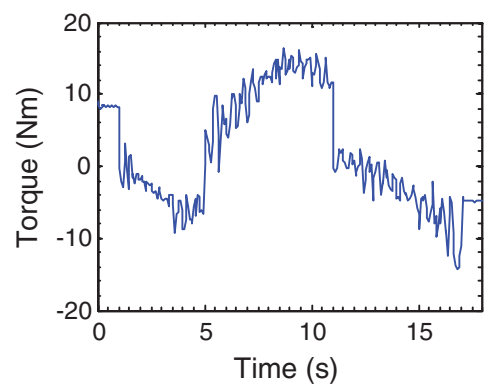

(c)

Fig. 11. Elbow flexion/extension (a) slow speed (b) medium, (c) fast speed. 

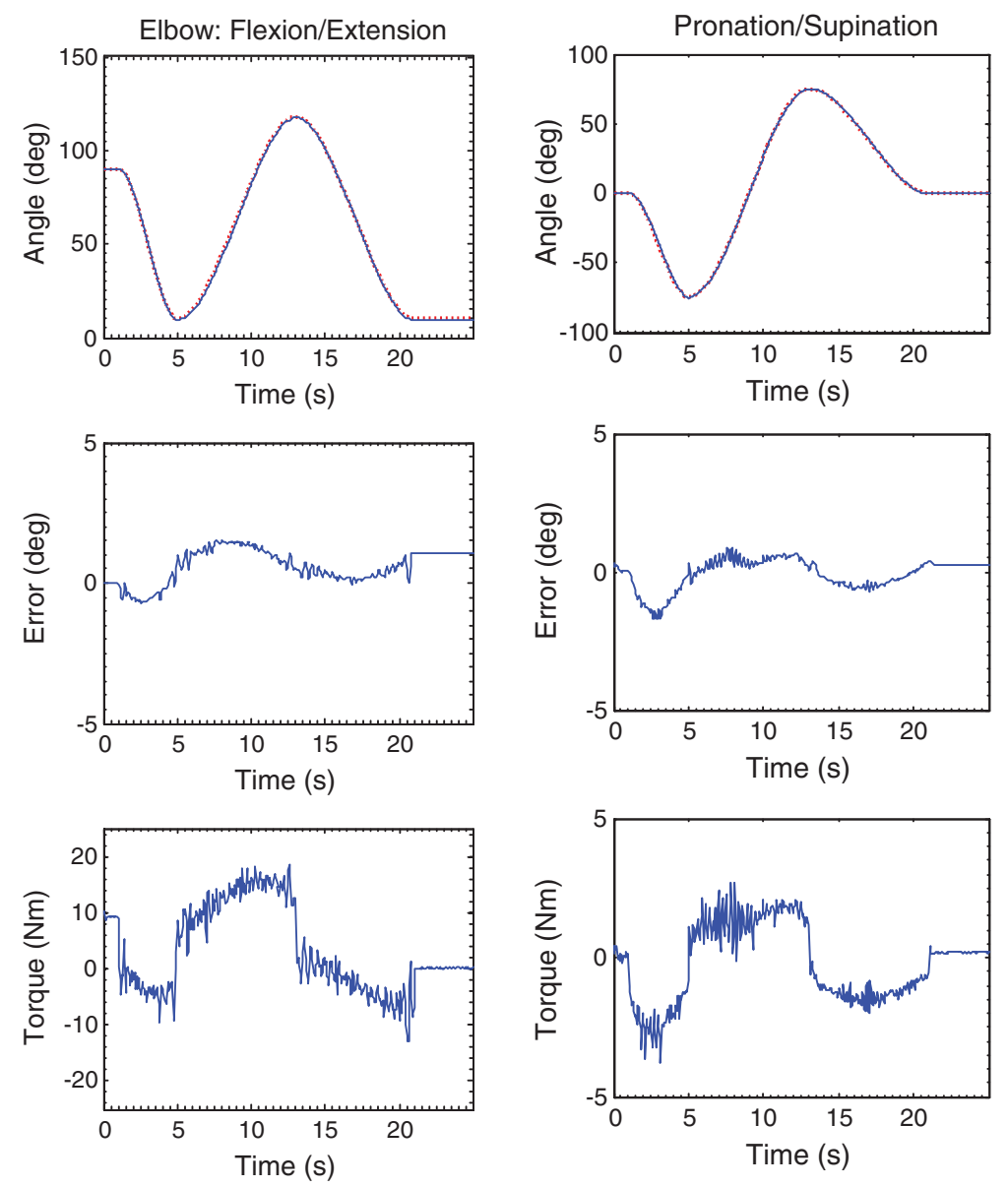

Fig. 12. Simultaneous movement of elbow and forearm (slow movement).

The signals are then filtered (second order filtering: $\left.\omega_{0}=30 \mathrm{rad} / \mathrm{s}, \zeta=0.9\right)$ prior to be sent to the controller. Filtering is important to eliminate high frequency or noisy data from the desired signals.

Control architecture for the MARSE-5 system is depicted in Fig. 10. The output of the controller is the joints torque commands. However, the torque commands are transferred to motor currents and finally to reference voltage as voltage value is the drive command for the motor drivers. Note that the controller (SMC) updates the torque commands at each $1.25 \mathrm{~ms}$ and is executed in RT-PC (left dotted circle, Fig. 10).

Furthermore, to realize the real time control of the MARSE-5, and also to ensure the right control torque command were sent to the joints (as well as the reference voltage commands for the drivers), a proportional-integral (PI) controller was added to minimize the differences in between desired and measured currents (i.e., the error command to PI controller). The PI controller runs 25 times faster than the torque control loop and is executed in FPGA of NI cRIO-9074. The current signals measured from the current monitor output of motor drivers are sampled at $0.1 \mathrm{~ms}$, and are then filtered with a second-order filter with a damping factor $\zeta=0.90$ and natural frequency $\omega_{0}=3000 \mathrm{rad} / \mathrm{s}$ prior to being sent to the PI controller (right dotted circle, Fig. 10). Note that the control gains used for the experiments were found by trial and error, and are as follows:

$$
\begin{aligned}
& \Lambda=\operatorname{diag}\left[\begin{array}{lllll}
10 & 10 & 10 & 10 & 10
\end{array}\right] \\
& K=\operatorname{diag}\left[\begin{array}{llllll}
200 & 100 & 230 & 400 & 2250
\end{array}\right]
\end{aligned}
$$



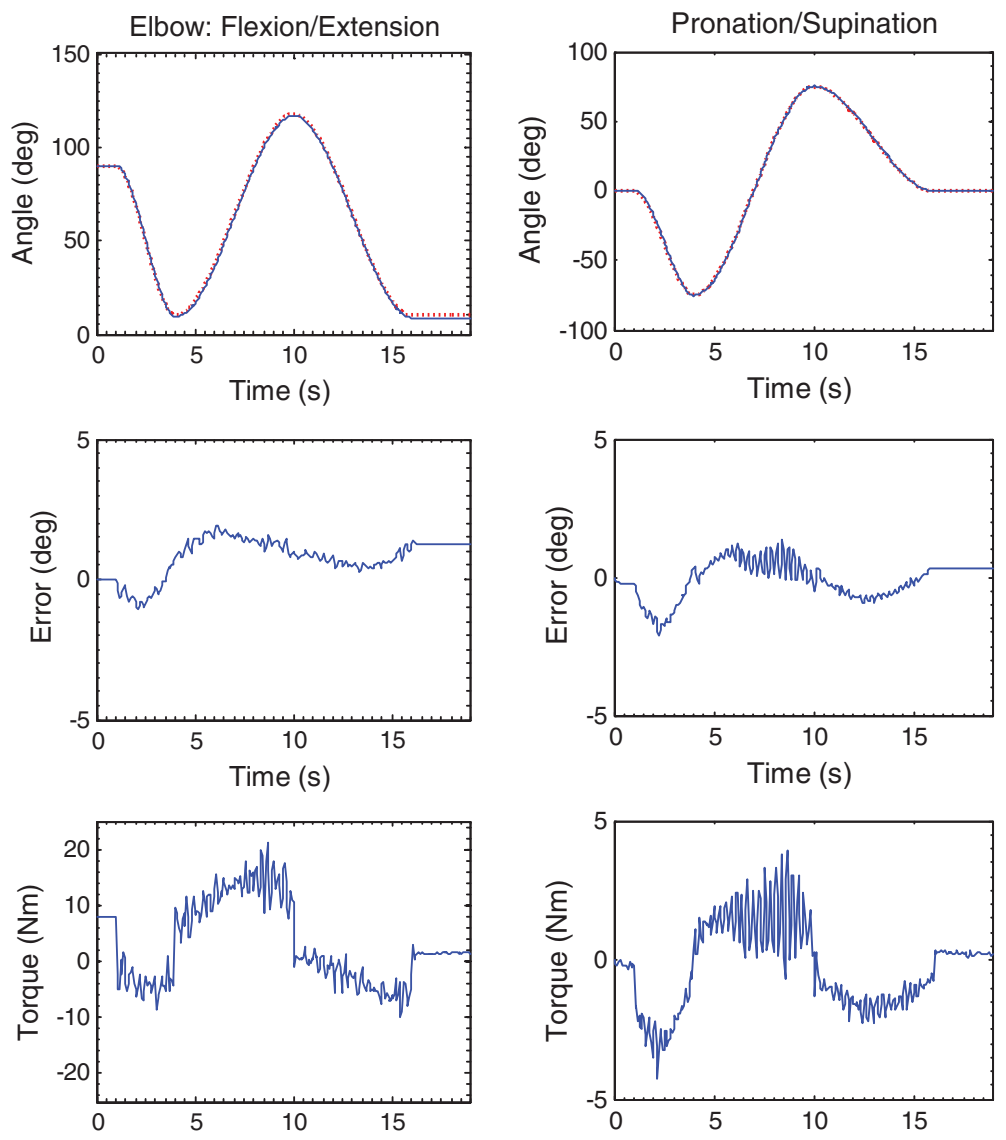

Fig. 13. Simultaneous movement of elbow and forearm (fast movement).

\subsection{Experimental results and analysis}

The experiments were conducted with subjects in a seated position. In experiments, the dynamic trajectory tracking performance of the MARSE-5 with SMC was evaluated. Note that typical upper-limb rehabilitation exercises [1] are considered for reference/desired trajectory and were generated using the cubic polynomial method [7]. The exercises used for this evaluation can be grouped under three categories; single joint movement, multi joint movements, and gravity weight balancing.

\subsubsection{Single joint movement}

Considering elbow joint movements are extensively used in performing daily upper-limb activities, an exercise involving elbow joint flexion and extension motion was chosen as a single joint movement exercise. This experiment were performed at three different speeds; slow $(0.3760 \mathrm{rad} / \mathrm{s})$, medium $(0.43 \mathrm{rad} / \mathrm{s})$ and fast $(0.502 \mathrm{rad} / \mathrm{s})$.

Figure 11a-c, show elbow joint flexion/extension motion for tracking conducted at slow, medium, and fast speed, respectively. The topmost plot of these figures compares the desired joint angles (or reference trajectories, dotted line) to measured joint angles (or measured trajectories, solid line). The 2 nd row of the plots shows the error as a function of time i.e., deviation between desired and measured trajectories. The generated joint torques corresponding to the trajectory is plotted in the bottom row. As shown from Fig. 11a the trial took $24 \mathrm{sec}$. to complete, whereas the experiment shown in Fig. $11 \mathrm{~b}$ took $21 \mathrm{sec}$., and the other one as depicted in Fig. 11c took $18 \mathrm{sec}$. to finish. It is obvious from the Fig. 11 that the performance of controller is excellent, since measured trajectories overlapped with the desired trajectories with tracking error limited to less than $1.5^{\circ}$. 

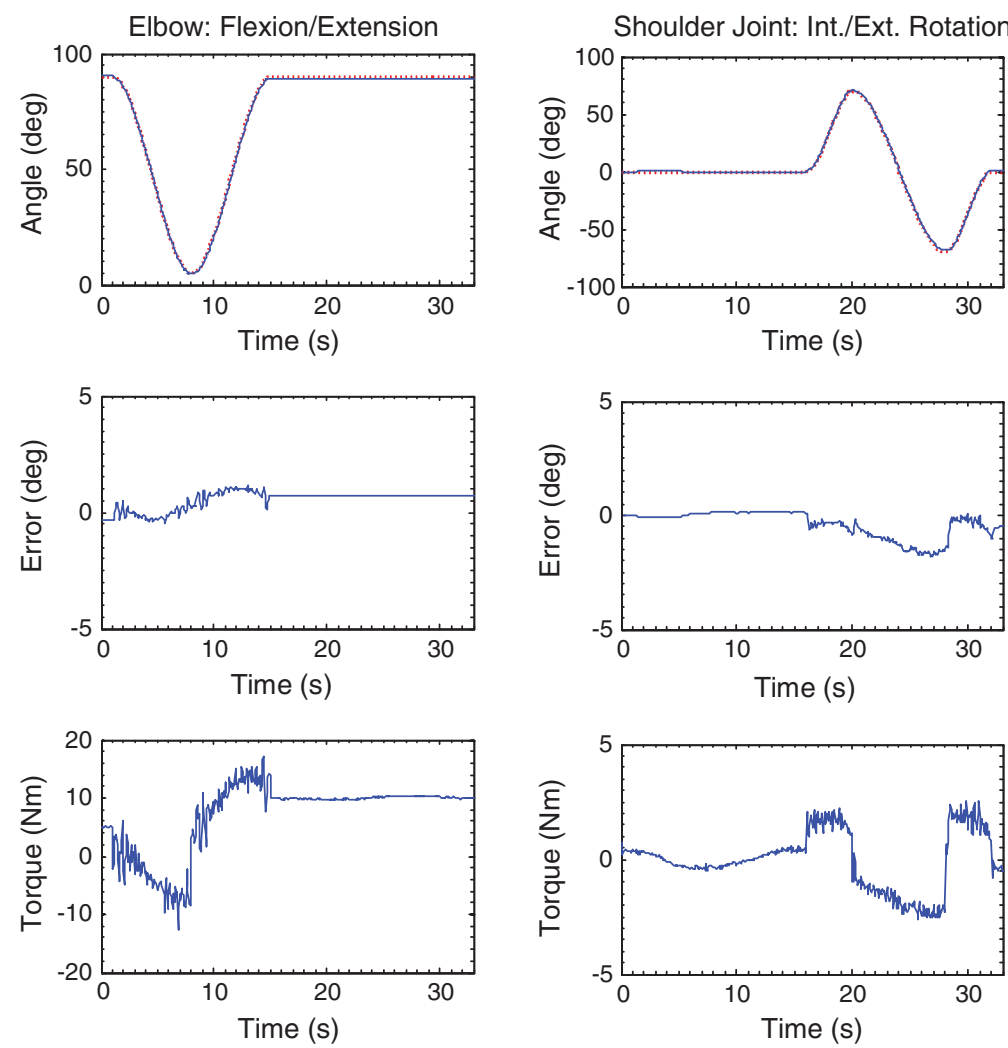

Fig. 14. Gravity weight balancing, a cooperative movement of elbow and shoulder joint internal/external rotation.

\subsubsection{Multi joint movements}

In this category the exercise involving multi joint movements was considered, where at least one joint movement is subject to gravity. Thus, a simultaneous movement of the elbow and the forearm were performed. Note that this exercise was conducted at two different speeds (slow and fast).

Figures 12 and 13 show the cooperative and simultaneous movement of forearm pronation/supination and elbow flexion/extension which were performed at slow and fast speed respectively. The objective of this task was to pronate the forearm from a neutral position, while simultaneously flexing the elbow from its initial position $\left(90^{\circ}\right)$ and then reversing the movement. The ability of the controller to track this movement is certainly obvious from these figures. As shown from Fig. 12, the trial took $25 \mathrm{sec}$. to complete, whereas the experiment shown in Fig. 13 took 19 sec. to complete. Also in these trials, SMC showed excellent tracking performance.

\subsubsection{Gravity weight balancing}

In this type of movement control, we evaluated the performance of the MARSE-5 in gravity weight balancing. For this, an experiment was set-up where the MARSE-5 is supposed to keep elbow flexed at $90^{\circ}$ while performing some other joint movement, e.g., shoulder joint internal/external rotation, or wrist joint flexion/extension. Note that weight of the forearm and hand is maximal when the elbow joint is at $90^{\circ}$.

Figure 14 demonstrates a co-operative movement of the elbow and shoulder joint internal/external rotation. As shown in Fig. 14, the exercise began with elbow extension followed by flexion up to $90^{\circ}$, thereafter maintaining that position while shoulder joint internal/external rotation was performed. Again, it was evident from the plots that tracking performance of the controller was excellent. It can also be seen from the Fig. 14 that steady state position error quite small (i.e., below $0.5^{\circ}$ ). 
Experimental results thus demonstrated the efficient performance of the control technique as well as the developed MARSE-5 regard to trajectory tracking. Note that these experiments are often used as an exercise to provide passive rehabilitation of the human upper-limb [1] and therefore could be performed with the developed MARSE-5.

\section{Conclusion}

A 5DoFs robotic exoskeleton (MARSE-5) corresponding to human shoulder, elbow and forearm has been developed to provide effective rehabilitation therapy for physically disabled people in deficits of upper-limb motion. In experiments, nonlinear sliding mode control technique was employed in the dynamic trajectory tracking that replicates passive arm movements, where it can be found that the developed $M A R S E-5$ can effectively provide passive rehabilitation therapy.

Future works include in developing a biological controller based on the skin surface electromyogram signals and the wrist force sensors signals to provide active assistance to the upper extremity movements [20].

Appendix-A

MARSE-5 at a Glance

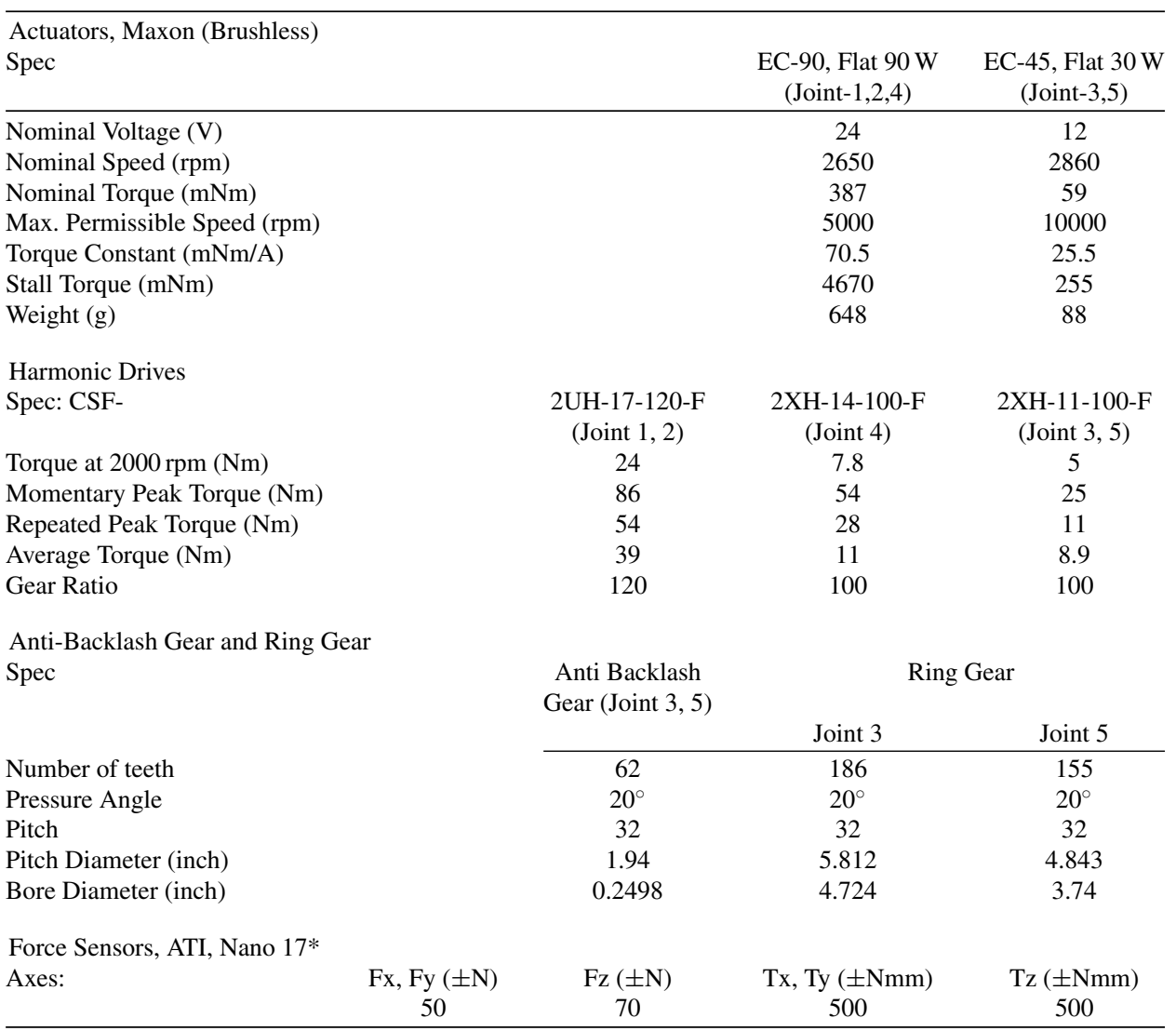

*A high linearity 6-axis force sensor (Nano 17, ATI) is instrumented underneath the wrist handle to measure the instantaneous reaction force. This signal will be used to actuate MARSE-5 in order to provide active assistanceour next step of research. 


\section{References}

[1] Physical Therapy Standards, in, Department of Rehabilitation Services, Brigham and Women's Hospital 2011. Available: http://www.brighamandwomens.org/Patients_Visitors/ pcs/rehabilitationservices/StandardsofCare.aspx, [Accessed: May, 1, 2012]

[2] S.J. Ball, I.E. Brown and S.H. Scott, MEDARM: A rehabilitation robot with $5 \mathrm{DOF}$ at the shoulder complex, in: 2007 IEEE/ASME International Conference on Advanced Intelligent Mechatronics (AIM2007), 4-7 Sept. 2007, IEEE, Piscataway, NJ, USA, 2007, pp. 284-289.

[3] S.W. Brose, D.J. Weber, B.A. Salatin, G.G. Grindle, H. Wang, J.J. Vazquez and R.A. Cooper, The role of assistive robotics in the lives of persons with disability, Am J Phys Med Rehabil 89 (2010), 509-521.

[4] C.G. Burgar, P.S. Lum, P.C. Shor and H.F.M. Van der Loos, Development of robots for rehabilitation therapy: The Palo Alto VA/Stanford experience, Journal of Rehabilitation Research and Development 37 (2000), 663-673.

[5] C. Carignan, J. Tang and S. Roderick, Development of an exoskeleton haptic interface for virtual task training, in: 2009 IEEE/RSJ International Conference on Intelligent Robots and Systems, IROS 2009, October 11, 2009-October 15, 2009, St. Louis, MO, USA, 2009, pp. 3697-3702.

[6] R. Colombo, F. Pisano, S. Micera, A. Mazzone, C. Delconte, M.C. Carrozza, P. Dario and G. Minuco, Robotic techniques for upper limb evaluation and rehabilitation of stroke patients, IEEE Transactions on Neural Systems and Rehabilitation Engineering 13 (2005), 311-324.

[7] J.J. Craig, Introduction to robotics: Mechanics and control, Pearson/Prentice Hall, Upper Saddle River, N.J., 2005.

[8] P.R. Culmer, A.E. Jackson, S. Makower, R. Richardson, J.A. Cozens, M.C. Levesley and B.B. Bhakta, A control strategy for upper limb robotic rehabilitation with a dual robot system, IEEE/ASME Transactions on Mechatronics 15 (2010), 575585.

[9] C.C. Dodson and F.A. Cordasco, Anterior glenohumeral joint dislocations, Orthop Clin North Am 39 (2008), vii, 507-518.

[10] A. Frisoli, F. Salsedo, M. Bergamasco, B. Rossi and M.C. Carboncini, A force-feedback exoskeleton for upper-limb rehabilitation in virtual reality, Applied Bionics and Biomechanics 6 (2009), 115-126

[11] P. Garrec, J.P. Friconneau, Y. Measson and Y. Perrot, ABLE, an innovative transparent exoskeleton for the upper-limb, in: 2008 IEEE/RSJ International Conference on Intelligent Robots and Systems, 22-26 Sept. 2008, IEEE, Piscataway, NJ, USA, 2008, pp. 1483-1488.

[12] R.A.R.C. Gopura, K. Kiguchi and L. Yang, SUEFUL-7: A 7DOF upper-limb exoskeleton robot with musclemodel-oriented EMG-based control, in: 2009 IEEE/RSJ International Conference on Intelligent Robots and Systems (IROS 2009), 11-15 Oct. 2009, Piscataway, NJ, USA, 2009, pp. 1126-1131.

[13] A. Gupta and M.K. O'Malley, Design of a haptic arm exoskeleton for training and rehabilitation, IEEE/ASME Transactions on Mechatronics 11 (2006), 280-289.

[14] N. Hamilton, W. Weimar and K. Luttgens, Kinesiology: Scientific basis of human motion, McGraw-Hill Higher Education, Boston, 2008.
[15] R.S. Hartenberg and J. Denavit, Kinematic Synthesis of Linkages, McGraw-Hill, New York, 1964.

[16] Hasan Hallaceli, Metin Manisali and I. Gunal, Does scapular elevation accompany glenohumeral abduction in healthy subjects? Arch Orthop Trauma Surg 124 ( 2004), 378381.

[17] K. Homma and T. Arai, Design of an upper limb motion assist system with parallel mechanism, in: Proceedings of the 1995 IEEE International Conference on Robotics and Automation. Part 1 (of 3), May 21, 1995-May 27, 1995, Nagoya, Japan, 1995, pp. 1302-1307.

[18] H. Kawasaki, S. Ito, Y. Ishigure, Y. Nishimoto, T. Aoki, T. Mouri, H. Sakaeda and M. Abe, Development of a hand motion assist robot for rehabilitation therapy by patient selfmotion control, in: 2007 IEEE 10th International Conference on Rehabilitation Robotics - ICORR'07, 12-15 June 2007, IEEE, Piscataway, NJ, USA, 2007, pp. 257-263.

[19] K. Kiguchi, K. Iwami, M. Yasuda, K. Watanabe and T. Fukuda, An exoskeletal robot for human shoulder joint motion assist, IEEE/ASME Transactions on Mechatronics 8 (2003), $125-135$.

[20] K. Kiguchi, M.H. Rahman, M. Sasaki and K. Teramoto, Development of a 3DOF mobile exoskeleton robot for human upper-limb motion assist, Robotics and Autonomous Systems 56 (2008), 678-691.

[21] H.I. Krebs, B.T. Volpe, M.L. Aisen and N. Hogan, Increasing productivity and quality of care: Robot-aided neuro-rehabilitation, Journal of Rehabilitation Research and Development 37 (2000), 639-652.

[22] R. Loureiro, F. Amirabdollahian, M. Topping, B. Driessen and W. Harwin, Upper limb robot mediated stroke therapy GENTLE/s approach, Autonomous Robots 15 (2003), 3551.

[23] J.A. Mehta and G.I. Bain, Elbow dislocations in adults and children, Clin Sports Med 23 (2004), 609-627.

[24] M. Mistry, P. Mohajerian and S. Schaal, An exoskeleton robot for human arm movement study, in: 2005 IEEE/RSJ International Conference on Intelligent Robots and Systems, 2-6 Aug. 2005, IEEE, Piscataway, NJ, USA, 2005, pp. 40714076.

[25] T. Nef, M. Guidali and R. Riener, ARMin III - arm therapy exoskeleton with an ergonomic shoulder actuation, Applied Bionics and Biomechanics 6 (2009), 127-142.

[26] J.C. Perry, J. Rosen and S. Burns, Upper-limb powered exoskeleton design, IEEE/ASME Transactions on Mechatronics 12 (2007), 408-417.

[27] C.A. Phillips, D.W. Repperger and T.L. Chelette, The acceleration-velocity relationship: Identification of normal and spastic upper extremity movement, Comput Biol Med $\mathbf{2 7}$ (1997), 309-328.

[28] G.B. Prange, M.J. Jannink, C.G. Groothuis-Oudshoorn, H.J. Hermens and M.J. Ijzerman, Systematic review of the effect of robot-aided therapy on recovery of the hemiparetic arm after stroke, J Rehabil Res Dev 43 (2006), 171-184.

[29] M.H. Rahman, T.K. Ouimet, M. Saad, J.P. Kenne and P.S. Archambault, Development and control of a wearable robot for rehabilitation of elbow and shoulder joint movements, in: IECON 2010 - 36th Annual Conference of IEEE Industrial Electronics, 7-10 Nov. 2010, Piscataway, NJ, USA, 2010, pp. 1506-1511. 
[30] M.H. Rahman, T.K. Ouimet, M. Saad, J.P. Kenne and P.S. Archambault, Dynamic Modeling and Evaluation of a Robotic Exoskeleton for Upper-Limb Rehabilitation, International Journal of Information Acquisition 8 (2011), 83-102.

[31] M.H. Rahman, M. Saad, J.P. Kenne and P.S. Archambault, Modeling and control of a 7DOF exoskeleton robot for arm movements, in: 2009 IEEE International Conference on Robotics and Biomimetics (ROBIO 2009), 19-23 Dec. 2009, Guilin, China, 2009, pp. 245-250.

[32] M.H. Rahman, M. Saad, J.P. Kenne and P.S. Archambault, Modeling and Development of an Exoskeleton Robot for Rehabilitation of Wrist Movements, in: 2010 IEEE/ASME International Conference on Advanced Intelligent Mechatronics (AIM 2010), 6-9 July 2010, Montreal, Canada, 2010, pp. 25-30.

[33] D.C. Reid, Sports Injury Assessment and Rehabilitation, New York, NY: Churchill Livingstone, 1992.

[34] J. Rosen, J.C. Perry, N. Manning, S. Burns and B. Hannaford, The human arm kinematics and dynamics during daily activities - toward a 7 DOF upper limb powered exoskeleton, in: Advanced Robotics, 2005. ICAR'05. Proceedings., 12th International Conference on, 2005, pp. 532-539.

[35] J.J.E. Slotine and W. Li, Applied nonlinear control, PrenticeHall, Englewood Cliffs, N.J., 1991.
[36] T.G. Sugar, H. Jiping, E.J. Koeneman, J.B. Koeneman, R. Herman, H. Huang, R.S. Schultz, D.E. Herring, J. Wanberg, S. Balasubramanian, P. Swenson and J.A. Ward, Design and control of RUPERT: A device for robotic upper extremity repetitive therapy, IEEE Transactions on Neural Systems and Rehabilitation Engineering 15 (2007), 336-346.

[37] C.D. Takahashi, L. Der-Yeghiaian, V. Le, R.R. Motiwala and S.C. Cramer, Robot-based hand motor therapy after stroke, Brain 131 (2008), 425-437.

[38] C.C. Tsao and M.M. Mirbagheri, Upper limb impairments associated with spasticity in neurological disorders, J Neuroeng Rehabil 4 (2007), 45

[39] R. Yupeng, P. Hyung-Soon and Z. Li-Qun, Developing a whole-arm exoskeleton robot with hand opening and closing mechanism for upper limb stroke rehabilitation, in: 2009 IEEE International Conference on Rehabilitation Robotics: Reaching Users \& the Community (ICORR), 23-26 June 2009, IEEE, Piscataway, NJ, USA, 2009, pp. 761-765.

[40] V.M. Zatsiorsky and V.N. Seluyanov, Mass and inertia characteristics of the main segments of the human body, in Biomechanics 8-B, Proceedings of the 8th International Congress of Biomechanics, Nagoya, Japan, 1983, pp. 11521159 . 

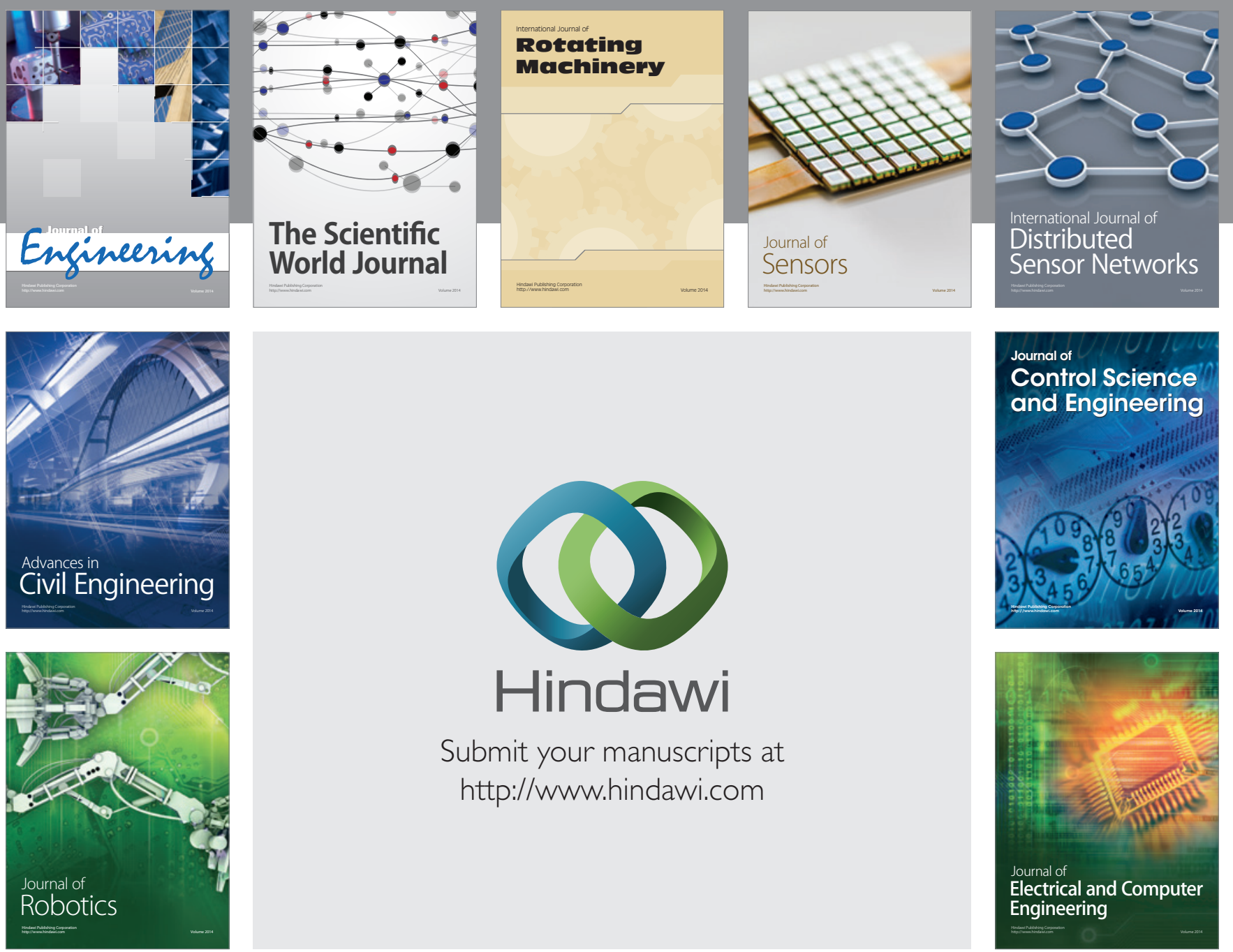

Submit your manuscripts at

http://www.hindawi.com
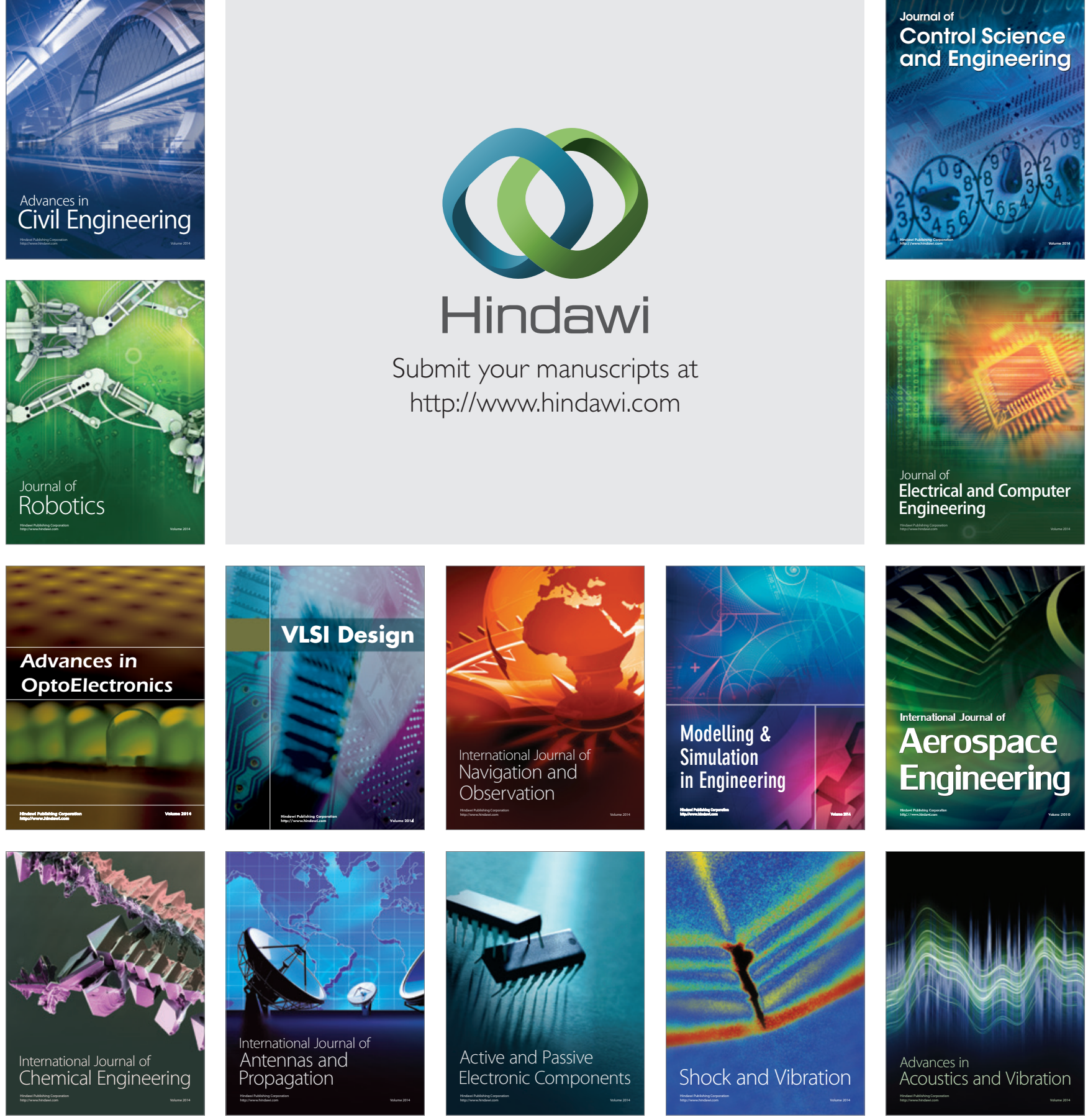